\title{
A survey of HK, HPt, and RR domains and their organization in two-component systems and phosphorelays proteins of organisms with fully sequenced genomes
}

Baldiri Salvado, Ester Vilaprinyo, Albert Sorribas, Rui Alves

Two Component Systems and Phosphorelays (TCS/PR) are environmental signal transduction cascades in prokaryotes and, less frequently, in eukaryotes. The internal domain organization of proteins and the topology of TCS/PR cascades play an important role in shaping the responses of the circuits. It is thus important to maintain updated censuses of TCS/PR proteins in order to identify the various topologies used by nature and enable a systematic study of the dynamics associated with those topologies. To create such a census, we analyzed the proteomes of 7609 organisms from all domains of life with fully sequenced and annotated genomes. To begin, we survey each proteome searching for proteins containing domains that are associated with internal signal transmission within TCS/PR: Histidine Kinase (HK), Response Regulator (RR) and Histidine Phosphotranfer (HPt) domains, and analyze how these domains are arranged in the individual proteins. Then, we find all types of operon organization and calculate how much more likely are proteins that contain TCS/PR domains to be coded by neighboring genes than one would expect from the genome background of each organism. Finally, we analyze if the fusion of domains into single TCS/PR proteins is more frequently observed than one might expect from the background of each proteome. We find 50 alternative ways in which the HK, HPt, and RR domains are observed to organize into single proteins. In prokaryotes, TCS/PR coding genes tend to be clustered in operons. $90 \%$ of all proteins identified in this study contain just one of the three domains, while $8 \%$ of the remaining proteins combine one copy of an HK, a RR, and/or an HPt domain. In eukaryotes, $25 \%$ of all TCS/PR proteins have more than one domain. These results might have implications for how signals are internally transmitted within TCS/PR cascades. These implications could explain the selection of the various designs in alternative circumstances. 
1 A survey of HK, HPt, and RR domains and their organization 2 in two-component systems and phosphorelays proteins of 3 organisms with fully sequenced genomes

6 Baldiri Salvado ${ }^{1}$, Ester Vilaprinyo ${ }^{1}$, Albert Sorribas ${ }^{1}$, Rui Alves ${ }^{1, *}$

10 1Departament de Ciències Mèdiques Bàsiques (CMB), Universitat de Lleida \& IRBLleida, Edifici

11 Recerca Biomèdica I,Av. Rovira Roure 80, 25198, Lleida, Spain

$12{ }^{*}$ Corresponding author

13 e-mail: ralves@cmb.udl.cat

14 Phone: +034973702425 


\section{Abstract}

17 Two Component Systems and Phosphorelays (TCS/PR) are environmental signal transduction

18 cascades in prokaryotes and, less frequently, in eukaryotes. The internal domain organization of

19 proteins and the topology of TCS/PR cascades play an important role in shaping the responses

20 of the circuits. It is thus important to maintain updated censuses of TCS/PR proteins in order to

21 identify the various topologies used by nature and enable a systematic study of the dynamics

22 associated with those topologies.

23 To create such a census, we analyzed the proteomes of 7609 organisms from all domains of life

24 with fully sequenced and annotated genomes. To begin, we survey each proteome searching

25 for proteins containing domains that are associated with internal signal transmission within

26 TCS/PR: Histidine Kinase (HK), Response Regulator (RR) and Histidine Phosphotranfer (HPt)

27 domains, and analyze how these domains are arranged in the individual proteins. Then, we find

28 all types of operon organization and calculate how much more likely are proteins that contain

29 TCS/PR domains to be coded by neighboring genes than one would expect from the genome

30 background of each organism. Finally, we analyze if the fusion of domains into single TCS/PR

31 proteins is more frequently observed than one might expect from the background of each

32 proteome.

33 We find 50 alternative ways in which the HK, HPt, and RR domains are observed to organize

34 into single proteins. In prokaryotes, TCS/PR coding genes tend to be clustered in operons. $90 \%$

35 of all proteins identified in this study contain just one of the three domains, while $8 \%$ of the

36 remaining proteins combine one copy of an HK, a RR, and/or an HPt domain. In eukaryotes,

$3725 \%$ of all TCS/PR proteins have more than one domain. These results might have implications 
38 for how signals are internally transmitted within TCS/PR cascades. These implications could

39 explain the selection of the various designs in alternative circumstances. 


\section{Introduction}

41 Historically, Two Component Systems and Phosphorelays (TCS/PR) have been considered as

42 primary environmental signal transduction cascades in prokaryotes (Wolanin, Thomason \&

43 Stock, 2002; Cheung \& Hendrickson, 2010). In TCS/PR, environmental signals regulate the

44 autophosphorylation state of a sensor histidine kinase. In TCS this sensor transfers its

45 phosphate to a response regulator, which will in turn directly regulate the relevant cellular

46 responses to the signal. The sensor and response regulator may be two independent proteins.

47 They may also be the same protein, containing independent domains that are responsible for

48 each of the two functions. In PR, additional phosphotransfer steps may happen before the

49 phosphate reaches the response regulator protein(s) that directly controls cellular responses

50 (Figure 1). PR are considered to be a main form of signal transduction in bacteria (Parkinson,

51 1993; Hoch, J. A., Silhavy, 1995). They are less frequently present in eukaryotes and absent in

52 animals (Chang et al., 1993; Maeda, Wurgler-Murphy \& Saito, 1994; Appleby, Parkinson \&

53 Bourret, 1996; Thomason \& Kay, 2000).

54 The mechanism of signal sensing in the various types of TCS/PR have been studied with

55 great detail and is reviewed elsewhere (Inouye \& Dutta, 2003; Simon, Crane \& Crane, 2007;

56 Gross \& Beier, 2012). Extensive and insightful reviews have also been published about the

57 topology (pattern of molecular interactions between the proteins in the cascade), crosstalk and

58 signal transmission in TCS/PR (Oka, Sakai \& Iwakoshi, 2002; Majdalani \& Gottesman, 2005;

59 Bekker, Teixeira de Mattos \& Hellingwerf, 2006; Laub \& Goulian, 2007; Szurmant, White \&

60 Hoch, 2007; Ortiz de Orué Lucana \& Groves, 2009; Krell et al., 2010; Buelow \& Raivio, 2010;

61 Szurmant \& Hoch, 2010; Hazelbauer \& Lai, 2010; Casino, Rubio \& Marina, 2010; Porter, 
62 Wadhams \& Armitage, 2011; Schaller, Shiu \& Armitage, 2011; Kobir et al., 2011; Seshasayee \&

63 Luscombe, 2011; Gross \& Beier, 2012; Jung et al., 2012; Podgornaia \& Laub, 2013; Fassler \&

64 West, 2013; Mascher, 2014), as well as about the domain structure and evolution of the

65 proteins involved in the cascades (Inouye \& Dutta, 2003; Catlett, Yoder \& Turgeon, 2003;

66 Galperin \& Nikolskaya, 2007; Cock \& Whitworth, 2007; Jenal \& Galperin, 2009; Whitworth \&

67 Cock, 2009; Kim et al., 2010; Wuichet, Cantwell \& Zhulin, 2010; Cheung \& Hendrickson, 2010;

68 Galperin, 2010; Perry, Koteva \& Wright, 2011; Seshasayee \& Luscombe, 2011; Capra \& Laub,

69 2012; Sheng et al., 2012; Attwood, 2013; Ortet et al., 2015).

There are several protein types and domains that nature uses in TCS/PR cascades. For

example, CHEW adapter proteins permit transmitting information about nutrient gradients to

the TCS system that regulates bacterial response to those gradients (Jones \& Armitage, 2015).

In another example, the PII protein regulates the activity of the TCS that responds to nitrogen depletion in the environment (Huergo et al., 2012). There are other cases where external proteins bind proteins from a TCS/PR cascade and modulate their stability (Salvado et al., 2012). These protein types are used in TCS/PR with specific biological functions and are not common to all TCS/PR cascades.

Nevertheless, there are four types of protein domains that are common to all TCS/PR cascades. Sensor domains, with wide sequence variability, are responsible for capturing the environmental changes and adjusting the activity of the cascade (Cheung \& Hendrickson, 2010;

81 Hazelbauer \& Lai, 2010). Irrespective of protein domain organization, signal transmission

82 within a TCS/PR circuit is done using histidine kinase (HK) domains, response regulator (RR) 83 domains, and/or histidine phosphotransfer (HPt) domains. These last three domains are 
84 responsible for internal signal transmission (IST) within the cascade and represent the focus of

85 the current work. Because they are common to all TCS/PR cascades, the results from our study

86 are generally applicable and do not depend on the specific environmental signal or biological

87 response mediated by the cascades.

88 Several examples demonstrate that the dynamic range and signal-response curve that a

89 given cascade might exhibit is closely related to the interactions between the various proteins

90 and to the organization of IST domains within each cascade protein (Alves \& Savageau, 2003;

91 Igoshin et al., 2004; Igoshin, Alves \& Savageau, 2008; Eswaramoorthy et al., 2010; Ray, Tabor \&

92 Igoshin, 2011; Salvado et al., 2012; Narula et al., 2012). For example, circuits where each IST

93 domain is in an independent protein are more likely to participate in cross-talk and branching is

94 more likely to occur in the signal transduction process (Catlett, Yoder \& Turgeon, 2003;

95 Seshasayee \& Luscombe, 2011). In addition, noise propagates differently in a cascade of

96 independent IST domain proteins than in a cascade where IST domains are found within the

97 same protein (Swain, 2004) (Figure 2). Also, TCS where phosphatases are involved in

98 dephosphorylating the response regulator protein may show hysteretic behavior. In contrast,

99 TCS where the sensor protein works both as phosphodonor and phosphatase for the response

100 regulator may only exhibit graded responses to changes in the signal (Igoshin, Alves \&

101 Savageau, 2008).

102 These and other examples (Alves \& Savageau, 2003; Süel et al., 2006; Kuchina et al.,

103 2011a,b) show that connectivity of the TCS/PR circuits and domain organization of the

104 proteins play an important role in shaping the responses of the cascades to their cognate

105 signals. It is thus important to maintain censuses of TCS/PR proteins in order to identify the 
106 various network topologies used by nature and enable a systematic study of the internal signal

107 transduction dynamics associated with those topologies. Information about such topologies

108 can be retrieved for a detailed analysis from several databases (Ulrich \& Zhulin, 2010; Finn et

109 al., 2014).

110 While MIST2 (Ulrich \& Zhulin, 2010) contains information about less than 3000

111 genomes, Pfam contains a few hundred sequences divided among the HK, RR, and HPt domain

112 families involved in TCS/PR cascades. Currently, at the NIH there are over 10000 fully

113 sequenced and annotated genomes that are freely accessible to the public. Because of this,

114 obtaining a more up to date census of the TCS/PR in these genomes is an important task that

115 we set out to do. We analyzed the TCS/PR proteins of 7609 organisms from all domains of life

116 with fully sequenced and annotated genomes. We focus on the IST domain families HK, RR, and

117 HPt of TCS/PR cascades. First, we survey the number of TCS/PR domains in each organism and

118 how these domains are arranged into individual proteins. Then, we find all different type of

119 operon organizations and analyze how much more likely are proteins that contain TCS/PR

120 domains to be coded by neighboring genes than one would expect from the genome

121 background. Finally, we analyze how the percentage IST domain fusion within TCS/PR proteins

122 changes among all analyzed genomes.

123 Our census finds that there are 50 alternative ways in which the $\mathrm{HK}, \mathrm{HPt}$, and RR

124 domains are observed to organize into single proteins. 90\% of all proteins identified in this

125 study contain just one RR or HK domain, while $8 \%$ of the remaining proteins combine one copy

126 of a HK, a RR, and/or a HPt domain. We also find that more than $25 \%$ of all TCS/PR eukaryotic

127 proteins have more than one domain. Our results are consistent with previous works and 
128 identify TCS/PR proteins in all non-animal phyla. Overall, our results set the stage for a

129 systematic study to compare the internal dynamic behavior of signal transduction associated

130 with each circuit topology in TCS/PR cascades.

\section{Material and Methods}

133

\section{Identification of proteins containing TCS/PR domains}

The fully annotated proteomes of 9961 organisms were downloaded from NCBI's genome database (January 2014 version). 2352 of these proteomes were eliminated because

137 they belonged to phages, virus, satellite DNA sequences, or organisms whose taxonomic

138 classification was still not fully resolved. The remaining 7609 proteomes belonging to 35 phyla

139 from Bacteria, 6 phyla from Archaea and 11 eukaryotic phyla (Supplementary Table 1) were

140 further analyzed in search for proteins containing domains of types HK, RR and HPt. These

141 domains are associated with IST in all TCS/PR cascades. Other protein domains (such as the

142 CHEW adaptor domain or the P2 protein from NRI/NRII, among many others) were not included

143 in the analysis because they are specific of certain TCS/PR cascades. The sensor domain of

144 TCS/PR cascade proteins was also not included due to its sequence variability. Thus, the results

145 from our study are general for all TCS/PR cascades.

146 We used PROSITE (http://prosite.expasy.org) to obtain a set of well curated sequences

147 that can be used as a seed to identify TCS/PR proteins in the relevant proteomes. We

148 downloaded a multiple alignment of all relevant ortholog sequences for each protein domain

149 (HK - PS50109 PROSITE Domain, RR - PS50110 PROSITE Domain and HPt - PS50894 PROSITE 
150 Domain) from PROSITE. We then used these three multiple alignments as a set of query

151 sequences for two independent searches. One was done using HMMER (Johnson, Eddy \&

152 Portugaly, 2010). For each multiple alignment downloaded from PROSITE, we built a profile

153 HMM using hmmbuild, and performed the search of the profile HMM against all proteomes

154 selected from the NCBI database using jackhmmer. The second search was done in parallel

155 using PSI-BLAST (Altschul et al., 1990) and the three multiple alignments downloaded from

156 PROSITE as a query. HMMER finds homologues that are more distantly related than those

157 found by BLAST.

158 We simultaneously use BLAST and HMMer because they have different sensitivities in

159 detecting sequence similarities. BLAST generates a higher number of false negatives, while

160 HMMer generates a higher number of false positives. By using both and filtering the results, we

161 hope to obtain a more precise picture of the conserved domains. In each search we queried the

1627609 proteomes in order to identify proteins with domains that are homologous to those used

163 as queries.

164 In addition, the consensus sequence was calculated for each domain (HK, RR and HPt)

165 independently. Using an in-house PERL script, the most common residue in each position was

166 identified for each of the three multiple alignments. This residue was taken as the consensus

167 value for that position in the corresponding protein domain. Subsequently the three consensus

168 sequences were used to search each proteome using PSI-BLAST (Altschul et al., 1990). In all

169 three searches, the hits selected were the ones with an e-value lower than $10^{-6}$ and with a

170 domain coverage of at least $80 \%$. 
After performing these three searches, a PERL script was also used to perform a fourth

172 text-mining search and identify the proteins that were annotated in each proteome as being

173 histidine kinases, sensory kinases, hybrid kinases, response regulators or histidine

174 phosphotransferases.

175 The results of the four searches were merged into a non-redundant set. A total amount

176 of 469421 proteins containing HK, HPt and/or RR domains were identified. This set was curated

177 in the following way:

$1781-$ First we manually looked at the annotation of the proteins to identify functions that are not

179 involved in TCS/PR cascades (e.g. serine kinase).

1802 - Then, we build a PERL script that automatically eliminates proteins annotated with those

181 functions from the list.

1823 - We finish by automatically comparing the proteins in the list and the number of proteins

183 containing terms related to TCS/PR cascades.

$1844-$ We repeat steps 1-3 until the number of proteins in the list and the number of proteins 185 containing only terms related to TCS/PR cascades are the same.

186 In this way, we semi manually identified 36169 proteins that were annotated as being

187 something other than a TCS/PR protein. These proteins were eliminated. Frequent protein 188 types found in the discarded set of proteins are serine/threonine kinases and several types of 189 regulatory transcription factors.

190 The remaining 433255 proteins were then reanalyzed and an additional set of 17727

191 proteins were found to be annotated as being hypothetical or partial proteins. For each of the

192 three domains, the set of 17727 hypothetical and partial proteins were aligned using Clustal X 
193 in order to identify the conserved histidine motif in the HK and HPt domains, and the

194 conserved aspartate residue in RR domains. Those sequences without a conserved histidine or

195 aspartate residue were eliminated from the data, leaving a grand total of 415525 annotated

196 proteins and 17724 partial/hypothetical proteins containing HK, RR and/or HPt domains.

197 A PERL script was developed to filter the curated data sets and determine both, the

198 domain composition of each protein and, when they belonged to the same organism, the

199 relative position of their corresponding genes with respect to each other in the genome.

200 Once we had identified all proteins containing HK, RR or HPt domains, and the relative

201 genomic position of their corresponding genes, we looked for all type of operons of TCS/PR

202 coding genes that occur in the organisms with fully sequenced genomes. For this purpose, we

203 performed a search of all genes coding HK, RR or HPt protein domains that are located in

204 consecutive positions on prokaryotic genomes. We assumed that they constitute a

205 transcription unit, although this may introduce a small error, as consecutive operons coding for

206 independent TCS/PR exceptionally exist. In our search, we allow the presence of a gap in the

207 operon, that is, a gene which does not encode any HK, RR or HPt domain, because this could be

208 a gene with regulatory functions in the operon.

209 The statistical treatment of data was carried out independently with and without taking

210 into account the hypothetical and partial proteins found. Both results are qualitatively the

211 same. In the Results section of this paper we give the results from the analysis of the set of

212 proteins without the hypothetical and partial proteins. The results of the whole set of proteins,

213 including hypothetical and partial proteins, are given as supplementary figures and tables. The 
214 sequence files for all domains are also available at

215 http://web.udl.es/usuaris/pg193845/Salvadoretal.html.

216

217

\section{Numerical and Statistical Data analysis}

219 To estimate how the clustering of the various TCS and PR proteins in a genome differed from

220 what one would expect by chance in the context of that genome, we took the following

221 approach. First, we calculated how frequently one would expect proteins containing TCS/PR

222 domains to be coded by neighboring genes in a genome if the order of genes was fully random,

223 given the total number of proteins in that genome, and the number of proteins involved in

224 TCS/PR cascades. The expected neighboring frequencies under this assumption can be

225 computed by Eqs. 1-6.In these equations $\mathrm{F}(\mathrm{P} 1 \leftrightarrow \mathrm{P} 2)$ represents the expected frequency of the

226 neighboring events in a genome for genes coding proteins of types $\mathrm{P} 1$ and $\mathrm{P} 2, \mathrm{n}_{\mathrm{RR}}$ represents

227 the number of proteins containing one RR domain in the proteome, $\mathrm{n}_{\mathrm{HK}}$ represents the number

228 of proteins containing one HK domain in the proteome, and $\mathrm{P}$ represents the total number of

229 proteins annotated to the proteome.

$230 \quad \mathrm{~F}(\mathrm{HK} \leftrightarrow \mathrm{RR})=\frac{\mathrm{n}_{R R}}{\mathrm{P}-1}+\frac{\mathrm{n}_{R R}}{\mathrm{P}-2}$

231 Eq. 1 represents the probability that a gene localized in position $\mathrm{j}$ of the genome is

232 located next to a gene coding for a protein that contains an RR domain, either in positions j-1 or

$233 \mathrm{j}+1$, if gene order is random in a genome. The first term of the sum represents the probability of

234 the presence of an RR gene in one of the two possible locations irrespective of its presence also

235 in the other genome location, and the second term is the probability of the presence of the RR 
236 gene in one of the two genome locations when it is not found in the other one. We note that

237 we are not calculating the probability of having a consecutive gene pair containing HK and RR

238 domains. Rather, for any genomic position $\mathrm{j}$, we ask what the probability of its neighboring a

239 gene containing an RR domain is. Eq. 1 gives a good estimation of this random probability, given

240 that the total number of protein coding genes is tens to hundreds of times larger than the

241 number of IST domain coding genes, and assuming that position $\mathrm{j}$ represents neither the first

242 nor the last genomic position. This expected RR neighboring frequency will be compared with

243 the actual fraction of HK genes that are found next to RR genes in order to study their genomic

244 distribution.

$245 \mathrm{~F}\left(\mathrm{HK} \leftrightarrow \mathrm{RR} \leftrightarrow \mathrm{HK}_{2}\right)=6 \times \frac{\mathrm{n}_{H K}}{\mathrm{P}-1} \times \frac{\mathrm{n}_{R R}}{\mathrm{P}-2}$

Eq. 2

246 Eq. 2 computes the probability of finding an RR gene and a second HK gene in the

247 genomic neighborhood of a given HK gene. Because these three consecutive genes can be

248 sorted in 6 different ways, we must multiply by 6 the probability of an individual neighboring

249 event. Again, note that we assume having an HK domain containing gene, and ask what the

250 probability of its neighboring genes containing additional HK and RR domains is.

$251 \mathrm{~F}\left(\mathrm{HK} \leftrightarrow \mathrm{RR} \leftrightarrow \mathrm{HK}_{2} \leftrightarrow \mathrm{RR}_{2}\right)=12 \times \frac{\mathrm{n}_{R R}}{\mathrm{P}-1} \times \frac{\mathrm{n}_{H K}-1}{\mathrm{P}-2} \times \frac{\mathrm{n}_{R R}-1}{\mathrm{P}-3} \quad$ Eq. 3

252 Similarly, in Eq. 3 we compute the probability that, considering that we have found a gene

253 containing an HK domain in a given place in the genome, we also find in consecutive genomic

254 positions around that HK gene location another HK gene and two RR genes, if gene organization

255 is random. These four genes can be sorted in 24 different ways, but we don't differentiate 
256 between the two RR genes and therefore there are only 12 possible spatial arrangements of

257 these series of four genes.

$258 \mathrm{~F}(\mathrm{HKRR} \leftrightarrow \mathrm{HK} \leftrightarrow \mathrm{RR})=6 \times \frac{\mathrm{n}_{H K}}{\mathrm{P}-1} \times \frac{\mathrm{n}_{R R}}{\mathrm{P}-2}$

259 In Eq. 4, the probability of the event is computed in exactly the same way as in Eq. 2.

$260 \mathrm{~F}(\mathrm{HKRRHPt} \leftrightarrow \mathrm{RR})=\frac{\mathrm{n}_{\mathrm{RR}}}{\mathrm{P}-1}+\frac{\mathrm{n}_{\mathrm{RR}}}{\mathrm{P}-2}$

Eq. 5

$261 \quad F(H K R R H K \leftrightarrow R R)=\frac{n_{R R}}{P-1}+\frac{n_{R R}}{P-2}$

Eq. 6

Eq. 5 and Eq. 6 compute the probability of finding and RR gene placed in the genome next to an HKRRHPt or an HKRRHK gene respectively, exactly in the same way as described above for Eq. 1.

Once these expected frequencies were computed using Eqs. 1-6, we calculated the odds ratios of the observed neighboring events with respect to the expected neighboring event. All numerical and statistical calculations were done using Mathematica (Wolfram, 1999).

\section{Statistical Models}

269 To analyze the relationship between the number of TCS/PR gene fusion events and the proteome size, we built a linear model that would better fit our data for \% of fused HK (RR, HPt)

271 domains vs. total number of HK (respectively, RR, HPt). We also built linear models of total

272 number of IST domains in an organism vs. total number of proteins in the proteome and

273 phylogeny (prokaryote, eukaryote). In other words, we fit the data to Eq. 7:

274 Number of IST domains $=\alpha_{1}$ (Total number of proteins in proteome $)+\alpha_{2}$ Phy $\log$ eny $+\varepsilon$ Eq. 7. 
275 In Eq. 7, the variable phylogeny can assume two values. If the organism is a prokaryote, the

276 variable has value 1 ; otherwise it has value 2 . An ANOVA analysis was used to determine

277 whether the coefficients for each control variable of the linear model are significantly different

278 from zero. If so, this implies that the variable is relevant in explaining the variation observed in

279 the dependent variable.

280 When fitting the data to the linear models we also calculated the $R^{2}$ and adjusted $R^{2}$ of the

281 models. $\mathrm{R}^{2}$ shows how well terms (data points) fit a curve or line; adjusted $\mathrm{R}^{2}$ also indicates how

282 well terms fit a curve or line, but adjusts for the number of terms in a model.

283

284 Results

285 Survey of proteomes containing proteins with domains involved in internal signal

286 transduction (IST) in TCS/PR cascades

287 Bacteria

288 Table 1 summarizes the full set of results for bacteria. Proteins with HK and RR domains are

289 present in the proteome of $100 \%$ of the species analyzed from the following bacterial phyla:

290 Aquificae, Chlorobi, Verrucomicrobia, Chloroflexi, Cyanobacteria, Deferribacteres,

291 Deinococcus-Thermus, Dictyoglomi, Acidobacteria, Nitrospirae, Planctomycetes,

292 Epsilonproteobacteria, Spirochaetes, Thermodesulfobacteria, and Thermotogae. In contrast,

293 proteins containing HK and/or RR domains were not identified in a small percentage of species

294 in the following phyla: Actinobacteria $-0.63 \%$ (4 out of 635 species surveyed), Bacteroidetes -

$2959.36 \%$ (22 out of 235 species), Firmicutes $-0.68 \%$ (14 out of 2066 ), Fusobacteria $-5.26 \%$ (2 
out of 38), Alphaproteobacteria - $3.55 \%$ (16 out of 451), Betaproteobacteria $-1.64 \%$ (6 out of

297 366), Deltaproteobacteria - 1.22 \% (1 out of 82), Epsilonproteobacteria $-0.24 \%$ (1 out of 410 ),

298 Gammaproteobacteria - 1.83 \% (41 out of 2246), Synergistetes - $9.09 \%$ (1 out of 11).

299 Interestingly, no proteins containing HK or RR domains were identified in most Tenericutes

300 species. Only 18 out of the 111 surveyed Tenericutes species have proteins with HK and RR 301 domains.

302 The percentage of species in each phylum with proteins containing HPt domains is lower

303 than the percentage of species with HKs and RRs, and ranges from less than $10 \%$ (Chlamydiae,

304 Tenericutes) to more than 90 \% (Deferribacteres, Acidobacteria, Nitrospirae, Planctomycetes,

305 Deltaproteobacteria, Epsilonproteobacteria, Gammaproteobacteria, Spirochaetes,

306 Thermodesulfobacteria, and Thermotogae). It should be noted that HPt domains are also used

307 by proteins that import PTS sugars (Clore \& Venditti, 2013), which means that not all HPt

308 domains we found are involved in PR or TCS signal transduction.

309 Archaea

310 Proteins with HK and RR domains were identified in the proteome of 154 out of 179

311 Euryarchaeota species, 9 of the 11 Taumarchaeota species and only 2 out of 51 Crenarchaeota

312 species surveyed. Proteins with HPt domains were identified in the proteome of 115

313 Euryarchaeota species and in 7 of the 11 Taumarchaeota species surveyed. No proteins

314 containing HK, RR, or HPt domains were identified in Nanoarchaeota, Nanohaloarcheota, and

315 Korarchaeota (Table 1).

316 Eukaryotes 
317 HK and RR domains were identified in the proteomes of 20 in 35 fungi species. 32 fungi species

318 contain proteins where the HPt-domain was identified. HK, HPt, and RR domains were

319 identified in the proteomes of the 2 eudicot species surveyed, but only HK and HPt, and not RR

320 domains, were identified in Oryza sativa.

321 There are only two surveyed protist phyla that contain proteins with IST domains. These

322 phyla are Euglenozoa and Amoeboflagellates. We analyzed five Euglenozoa species. Out of

323 these, only Leishmania donovani and Leishmania major contain proteins with HK and RR IST

324 domains. These domains are always found in separate proteins. Interestingly, only one RR

325 domain containing protein was identified in each of the two species. Surprisingly, only HK

326 domains were identified in proteins from Leishmania_infantum and Trypanosoma_brucei. No

327 IST domains were identified in Leishmania_braziliensis. In Amoeboflagellates, only

328 Dictyostelium discoideum has proteins containing IST domains in its proteome. The HK domain

329 was only identified in hybrid HKRR, HKRR1RR2, or HKRR1HK2RR2 proteins. In contrast, RR

330 domains also appear in proteins where no other IST domains are identified.

331 No HK, RR, or HPt domains were found in animal proteomes in the context of TCS/PR

332 cascades.

333

334 Percentage of proteins with HK, RR or HPt domains in the surveyed proteomes

335 For simplicity, hereafter we shall refer to proteins containing IST domains typical from TCS/PR

336 cascades as TCS/PR proteins. On average, between 1 and $2 \%$ of a prokaryotic proteome is

337 composed of TCS/PR proteins (mean $=1.37 \%$ ). In contrast, when an eukaryotic proteome

338 contains TCS/PR proteins, they account for between $0.05 \%$ and $0.2 \%$ of the entire proteome 
339 (mean $=0.11 \%)$. In bacteria, Deltaproteobacteria is the group with the highest average

340 percentage of TCS/PR proteins (Figure 3). In contrast Tenericutes and Chlamydiae almost tie

341 with the lowest average percentage of TCS/PR proteins (Figure 3).

342 It has been observed in previous analyses that the number of proteins containing IST

343 domains associated with TCS/PR cascades increases almost quadratically with the number of

344 total proteins in a proteome (Ulrich, Koonin \& Zhulin, 2005; Galperin, Higdon \& Kolker, 2010).

345 We further wanted to assess if this dependency is significantly different between eukaryotes

346 and prokaryotes. To do so we fit the data to the linear model described by Eq. 7. An ANOVA

347 analysis shows that phylogeny is important in explaining the variation in total number of IST

348 domains found in a proteome $\left(\mathrm{p}<10^{-25}\right)$. Because of this we divided the dataset in prokaryotes

349 and eukaryotes, and fit each dataset to the linear model shown in Figure 4. We find that the

350 fraction of variability in number of IST domains explained by proteome size in eukaryotes

351 doubles that of prokaryotes. This suggests that the number of IST domains could evolve

352 differently in prokaryotic and eukaryotic organisms.

353

354 Survey of TCS/PR protein types

355 We find fifty unique types of TCS/PR proteins, when it comes to IST domain organization within

356 a single polypeptide chain. These unique types of TCS/PR proteins are shown in Table 2, sorted

357 by abundance. In that table, the protein identifier describes the type of IST domain (HK, HPt, or

$358 \mathrm{RR}$ ) and the number describes how many domains of a given IST type are found in each

359 protein. Hereafter we shall refer to proteins containing only one HK IST domain as HK protein 
360 type, proteins containing one HK domain and one RR domain as HKRR protein type, and so on 361 and so forth.

362 Overall, all phyla where IST domains associated with TCS/PR cascades were identified

363 have RR and HK protein types, with the exception of Monocots, which lack RR domains. HKRR

364 protein type (also known as hybrid HK) is present in all phyla where TCS/PR proteins were 365 identified, except in Aquificae, Tenericutes, Chlamydiae, and Crenarchaeota (Supplementary

366 Table 2). Together, HK, RR, and HKRR proteins represent $94 \%$ of all TCS/PR proteins that were 367 identified.

368 In prokaryotes, RR or HK protein types are the most abundant. Together, they represent 369 more than $90 \%$ of all TCS/PR proteins found in the genomes of many organisms

370 (Supplementary Table 2). HKRR represent the third most abundant type of TCS/PR protein, 371 oscillating between less than $1 \%$ (Firmicutes) and more than 10\% (Cyanobacteria) of all TCS/PR

372 proteins (Supplementary Table 2). The remaining protein types (HPt, HKRRHPt, $\mathrm{HK}_{1} R R H K_{2}$, $\left.373 \mathrm{HKRR}_{1} \mathrm{HPtRR}_{2}, \mathrm{HK}_{1} \mathrm{RR}_{1} \mathrm{HK}_{2} \mathrm{RR}_{2}, \ldots\right)$ range from less than $1 \%$ to $5 \%$ of all TCS/PR proteins 374 identified in a phylum. Of these less abundant protein types, the three-domain HKRRHPt 375 protein is more abundant than $\mathrm{HK}_{1} \mathrm{RRHK}_{2}$. The HPt domain is more frequently found in 376 combination with other IST TCS/PR protein domains than alone in a protein, with the exception 377 of Firmicutes, Tenericutes, Actinobacteria, Bacteroidetes and Spirochaetes. We also observe 378 that $H K R R_{1} H P t R R_{2}$ is more abundant than $H_{1} R_{1} R_{1} H K_{2} R R_{2}$ (Supplementary Table 2).

379 The relative abundances of proteins containing IST domains associated with TCS/PR 380 cascades in eukaryotes are different from those of prokaryotes. In broad terms, HK and RR 381 protein types tend to make for a smaller fraction of TCS/PR proteins in eukaryotes than in 
382 prokaryotes, while the opposite is observed for HKRR proteins. Another clear distinction

383 between prokaryotes and eukaryotes refers to HPt-containing proteins: HPt protein type 384 represents more than $10 \%$ of all TCS/PR proteins in eukaryotes. In prokaryotes, except in

385 Tenericutes, HPt proteins typically account for less than $1 \%$ of TCS/PR proteins. Moreover, no 386 HKRRHPt or HKRR ${ }_{1} \mathrm{HPtRR}_{2}$ protein types were found in eukaryotes (Supplementary Table 2).

387 Among protists, Euglenozoa proteomes contain mostly HK protein type, although HKRR

388 type is the most abundant in $D$. discoideum (Amoeboflagellate). There are cases of inactive HK 389 domains that have lost their histidine. When identified, these proteins were eliminated from 390 the analysis as described in Methods. However, there is always the possibility that some such

391 proteins have passed our filters. To control for that possibility we created a multiple alignment

392 of the Euglenozoa HK proteins. We found that the HK domains contained the conserved 393 histidine motif that is needed for HK signal transduction. Hence, these proteins could be active

394 HK proteins. Furthermore, if we lower our e-value for cut-off to $10^{-4}$, many of these proteins

395 will also be flagged as containing RR domains with conserved aspartate residues, suggesting

396 that such proteins could be HKRR types with a high degree of sequence divergence from other

397 HKRR proteins we identified. Thus, the HK proteins in this clade might either be hybrid HKs or

398 be active in a context that does not involve a TCS/PR cascade. TCS/PR proteins are almost

399 absent in Alveolates. In the fungi phyla (Table 1), HKRR is the most abundant protein type in

400 Basidiomycetes, making up for almost $50 \%$ of total TCS/PR proteins. In contrast, RR, HK and

401 HPt protein types are relatively more abundant than HKRR protein type in Ascomycetes. A

402 remarkable result in fungi is the relative abundance of $H K_{1} R R H K_{2}$ and $H K_{1} R R_{1} H K_{2} R R_{2}$, which are 
403 much more frequent in eukaryotes (above $10 \%$ ) than in prokaryotes. In plants, RR is the most

404 abundant protein type in Eudicots, making for about $60 \%$ of all TCS/PR proteins.

405

406 Distribution of genes coding for TCS/PR protein types in the genomes

407 Previous surveys found that many of the TCS/PR proteins are mostly organized in 408 operons and/or regulons in prokaryotes (Alm, Huang \& Arkin, 2006; Williams \& Whitworth, 409 2010; Galperin, 2010; Galperin, Higdon \& Kolker, 2010). Consistent with this, we find that 410 between $60 \%$ and $90 \%$ of genes containing HK domains are neighbors to genes containing RR 411 domains. Exact percentages depend on the phylum, but below $20 \%$ of the total prokaryotic HK 412 coding genes are orphan, that is, they are not neighboring any other gene coding for a protein 413 that contains at least one IST domain. We also have found some clusters of genes coding HK, 414 RR or HPt domains in eukaryotes, but all of them are a succession of genes with identical 415 domain composition. Although the existence of operons has been reported in the eukaryote $C$. 416 elegans (Blumenthal, Davis \& Garrido-Lecca, 2015), the gene clusters identified in our search 417 have independent promoters.

418 Altogether, we found 530 different types of gene clusters coding for TCS/PR proteins.

419 We now briefly describe these results, shown in Supplementary Table 9.

421 Neighborhood analysis for HK and RR protein types

422 In most prokaryotes neighboring genes coding for HK and RR protein types are between 50 and 423100 times more frequent than one might expect by chance alone. In some species, this 424 frequency is even higher (Supplementary Figure 1, Supplementary Table 3). Several phyla have 
425 a small percentage of species containing only orphan HK and RR protein types in their genomes

426 (20 out of 2066 species in Firmicutes, 2 out of 635 in Actinobacteria, 6 out of 235 in

427 Bacteroidetes, 11 out of 2246 in Gammaproteobacteria, 48 out of 451 in Alphaproteobacteria,

4287 out of 366 in Betaproteobacteria, 4 out of 108 in Chlamydiae, 3 out of 118 in Cyanobacteria

429 and 9 out of 179 in Euryarchaeota). Most of these species have a number of TCS/PR proteins

430 below the average of their phylum.

431

432 Neighborhood analysis for HK-RR-HK2

433 Approximately $20 \%$ of all prokaryotic species have HK-RR-HK2 consecutive genes in their

434 genomes at least 10 (and sometimes 50) times more frequently than one might expect by 435 chance alone. Conversely, the frequency of this gene neighborhood organization is what one 436 would expect by chance alone in the remaining $80 \%$ prokaryotic species (Supplementary Table 437 4).

439 Neighborhood analysis for HK-RR-HK2-RR2

440 In most prokaryotic phyla, between $10 \%$ and $60 \%$ of species have $H K-R R-H K 2-R R 2$ genes at

441 least 100 times more frequently than one would expect by chance alone (Supplementary Table $4425)$.

444 Neighborhood analysis for HKRR-HK2-RR2

445 In the majority of prokaryotic species, genes coding for proteins of type HKRR have no 446 neighboring genes coding for proteins of types HK or RR. Nevertheless, in more than $20 \%$ of 
447 the species of some prokaryotic phyla, such as Proteobacteria or Spirochaetes, genes coding

448 for HKRR-protein type are neighbors to genes coding for HK or RR protein type with a

449 frequency more than 100 times higher than expected by chance alone (Supplementary Table 450 6).

452 Neighborhood analysis for HKRRHPt next to RR2

453 In most of the prokaryotic species where HKRRHPt protein types are present, the observed

454 frequency of HKRRHPt-RR genetic neighborhoods is between 10 and 50 times more frequent

455 than one would expect by chance alone (Supplementary Table 7).

457 Neighborhood analysis for HK1RRHK2 next to RR2

458 In prokaryotes, $\mathrm{HK}_{1} \mathrm{RRHK}_{2}$ is a scarce protein, present only in a few species (Table 2). If present, 459 it is located in the genome next to a RR protein type on average $31 \%$ of the times (Table 3). In

460 Gammaproteobacteria, $\mathrm{HK}_{1} \mathrm{RRHK}_{2}$ is present only in 28 out of 2246 species surveyed, and in 9

461 of these 28 species, the observed frequency of $\mathrm{HK}_{1} \mathrm{RRHK}_{2}$ genes placed in the chromosome 462 next to RR genes is more than 100 times higher than the random expected frequency 463 (Supplementary Table 8).

464

465 Gene fusion of TCS/PR proteins

466 Gene fusion events

467 The number of gene fusion events observed in a genome is expected to be proportional to 468 genome size, in a model for neutral evolution of protein domain fusion (Durrens, Nikolski \& 
469 Sherman, 2008; Whitworth \& Cock, 2009). Thus, if gene fusion events in the case of HK and RR

470 are random one would expect that the linear model that would best fit the data for $\%$ of fused

471 HK (RR, HPt) domains vs. total number of HK (respectively, RR, HPt) domains has slope zero. In

472 contrast, if these events are favored, the slope of that model should be positive, and if the

473 events are disfavored, that slope should be negative.

474 We analyze fusion events of IST domains associated with TCS/PR cascades in the

475 individual phyla by creating a linear model of percentage of fused HK (or RR) domains as a

476 function of the total number of HK (or RR) domains in the genome and calculate the likelihood

477 that the slope is different from zero. The results are shown in Table 4. We find that the

478 percentage of fused HK (or RR) domains increases with the number of HK (or RR) domains in

479 the genomes. This is consistent with a positive selection for fused HKRR proteins.

480

481 Discussion

482 Scope, caveats, and limitations of our analysis

483 In this work we analyze the distribution and prevalence of different types of TCS/PR proteins in

4847609 organisms belonging to 52 phyla. These proteins are responsible for sensing and

485 adequately regulating the cellular responses to environmental cues. To date, this is the largest

486 survey of TCS/PR proteins we are aware of. We confirm that these proteins are predominantly

487 prokaryotic, although they are also present in many eukaryotic phyla. However, functional

488 TCS/PR cascades appear to be absent in animals. This is also consistent with previous findings

489 (Attwood, 2013). 
An important feature in this study is that we include all organisms with fully sequenced

491 and annotated genomes in our analysis. For example, on the order of one thousand Escherichia

492 coli strains are included in our analysis. This would clearly bias any deletion/duplication or

493 horizontal gene transfer study of TCS/PR proteins that one might make in the full dataset.

494 However, considering all strains and subspecies in our analysis is fundamental for identifying

495 extremely low-frequency unique IST domain and operon organization types.

\section{Identifying unique types of IST domain organization in TCS/PR cascades}

498 The main goal of this analysis is to identify the unique types of organization for IST domains in 499 proteins of TCS/PR cascades. In addition we also perform a less thorough identification of operon organization for TCS/PR proteins. This study was independently made in two ways: first,

501 we eliminate all proteins annotated as hypothetical or partial. Subsequently we include such

502 proteins in the analysis. The results for the analysis that include the hypothetical and partial

503 proteins can be found as a ZIP supplementary file (Supplementary Appendix 1). Results are

504 qualitatively similar in both cases, and the raw sequences in FASTA format can be downloaded

505 from http://web.udl.es/usuaris/pg193845/Salvadoretal.html.

Our analysis identifies 50 unique types of TCS/PR proteins, when it comes to intra

507 protein IST domain organization. The most frequent types of proteins with fused IST domains

508 are the hybrid histidine kinases, a design with one HK and one RR protein domains fused in a

509 single protein. This organization has been observed in most of the eukaryotic PRs that have

510 been well characterized genetically and biochemically (for example the SIn1p-Ypd1p-Ssk1p

511 pathway in S. cerevisiae (Maeda, Wurgler-Murphy \& Saito, 1994) or the ETR1 system in A. 
512 thaliana (Chang et al., 1993)). It is also present in some prokaryotic systems (for example, the

$513 \mathrm{RcsC} /$ YojN/RcsB pathway, involved in the regulation of capsular polysaccharide synthesis in $E$.

514 coli (Takeda et al., 2001), and the Lux pathway regulating bioluminescence in $V$. harveyi

515 (Freeman \& Bassler, 1999)). Another relatively frequent type of IST domain organization is

516 when one HK, one HPt, and one RR domain are found within a single protein. Such proteins are

517 called unorthodox histidine kinase or tripartite HK. Some examples of systems with this design

518 are: BvgS-BvgA (Uhl \& Miller, 1996), EvgS/EvgA (Bock \& Gross, 2002), ArcB/ArcA (Georgellis,

519 Lynch \& Lin, 1997), TorS/TorR (Bordi et al., 2003), BarA/UvrY (Sahu et al., 2003), TodS/TodT

520 (Silva-Jiménez, Ramos \& Krell, 2012) and GacS/GacA (Sahu et al., 2003).

521 We also identify 530 unique types of possible operons in prokaryotes and some

522 eukaryotes, such as ascomycetes and eudicots (Supplementary Table 9). This variety will be

523 used in subsequent works to infer naturally occurring variations in the pattern of regulatory

524 interactions between the proteins involved in TCS/PR networks. For example, if we find a gene

525 cluster formed by one HK and two RR coding genes, we can infer that the signaling pathway has

526 a branching point in which the HK phosphorylates both RR. This alternative circuitry is

527 important because it has been proved that network architecture affects network dynamics and

528 can define the operational limits of the system in a way that is independent of the specific

529 biological processes being regulated (Alves \& Savageau, 2003; Igoshin, Alves \& Savageau, 2008;

530 Cağatay et al., 2009; Tiwari et al., 2010; Salvado et al., 2012).

531 We have no way of identifying TCS/PR cascades at the regulon level using only sequence

532 data. Many examples for this type of organization exist, such as the Kin-SpoO pathway 533 (Burbulys, Trach \& Hoch, 1991). 
535 also other protein domain that are involved in TCS/PR signal transduction? By focusing on these 536 domains and their organization, our results set the stage for an analysis of general dynamics

537 organization principles in the internal transmission of signals within TCS/PR cascades. The 538 organization of IST domains, either within a protein or within an operon, plays an important 539 role in determining the dynamics of the signal transmission in a cascade (Alves \& Savageau, 540 2003; Igoshin, Alves \& Savageau, 2008; Ray \& Igoshin, 2010; Narula et al., 2012). Hence, that

541 organization is likely to be the subject of natural selection. Had we included other types of 542 domains, we would be also analyzing aspects of the input and output of the cascades that are 543 case specific and not general to all cascades of a given type.

\section{Some physiological, phylogenetic and evolutionary considerations}

546 In prokaryotes, approximately $90 \%$ of all PR proteins have only one HK domain or one RR 547 domain (Table 2 and Supplementary Table 2), and most of the genes encoding these proteins 548 are located in the chromosome next to other PR/TCS genes, forming operons. In contrast to 549 this, in eukaryotes proteins of types HK and RR are less common, and genes encoding these 550 proteins are never located next to other TCS/PR genes in the species surveyed. On the other 551 hand, in eukaryotes there is a higher fraction of TCS/PR proteins containing a combination of

552 the HK and RR domains (the HPt domain was not found in these eukaryotic multi domain 553 TCS/PR proteins), such as HKRR, $\mathrm{HK}_{1} \mathrm{RRHK}_{2}$ and $\mathrm{HK}_{1} \mathrm{RR}_{1} \mathrm{HK}_{2} \mathrm{RR}_{2}$. This implies that TCS/PR signal 554 transduction in eukaryotes is in principle less prone to cross-talk and noise, as the signal is 
555 internally transmitted within the same peptide chain (Tiwari et al., 2010; Tiwari \& Igoshin, 556 2012).

557 Our analysis confirms the ad hoc observation that coordinated expression of IST 558 domains and/or TCS/PR proteins involved in the same cascade is frequent. We also quantify 559 how much more frequent this coordinated expression is with respect to what one would expect 560 by chance alone. Although this is not unexpected (Price, Arkin \& Alm, 2006; Alm, Huang \& 561 Arkin, 2006; Ray \& Igoshin, 2012; Tiwari \& Igoshin, 2012), to our knowledge, such quantification

562 had not been done before on such a large dataset.

563 This suggests that alternative IST regimes might be favored by evolution in prokaryotic 564 or eukaryotic TCS/PR cascades. This can be inferred from the fact that the three types of gene 565 expression coordination (regulon, operon, or gene fusion) imply different characteristics when

566 it comes to internal signal transmission within the cascade. In general, fused genes will have a

567 lower level of noise in signal transduction, followed by genes coded in the same operon, and 568 with genes coded in the same regulon permitting the highest level of noise to enter the signal 569 transduction process (Ray \& Igoshin, 2012).

570 Why is this so? TCS/PR proteins whose expression is coordinated either at the regulon or 571 operon levels are potentially translated in different amounts. RR proteins typically are orders 572 of magnitude more abundant than HK proteins (Igoshin, Alves \& Savageau, 2008). This leads to 573 a type of signal transduction where amplification of the signal can be high, as many RR 574 molecules can be modified by a single HK protein. In contrast, in hybrid kinases where the HK 575 and RR domains are fused in the same protein, the ratio of HK/RR domains is one to one. This 576 means that each HK domain will likely only phosphorylate one RR domain. Moreover, 
577 independent HK protein types might also be leakier, phosphorylating non-cognate RRs.

578 Similarly, independent RR protein types can be more prone to phosphorylation by non-cognate

579 sources. Such non-cognate phosphorylation events are physically harder to achieve in HKRR

580 protein types. Thus, proteins with fused TCS/PR domains represent a design that will on

581 average transduce signals with smaller amplification, but higher fidelity than TCS/PR cascades

582 composed only of proteins with individual TCS/PR domains.

583 Taking these considerations into account, one might think that maximization of internal

584 signal amplification is likely to be an important selective pressure for the evolution of TCS/PR

585 cascades in prokaryotes, while fidelity of internal signal transmission appears to be a more

586 important selective pressure for the evolution of TCS/PR cascades in eukaryotes. These two

587 functional requirements for IST in TCS/PR cascades are generic and independent of more

588 specific pressures, such as the type of signal they transduce, whether the organism is uni- or

589 multi-cellular, or other similar considerations (Alm, Huang \& Arkin, 2006; Laub \& Goulian,

590 2007; Williams \& Whitworth, 2010; Galperin, Higdon \& Kolker, 2010; Capra \& Laub, 2012;

591 Podgornaia \& Laub, 2013). If and why amplification and fidelity of internal signal transmission

592 are indeed shaping the general organization of TCS/PR cascades is a matter to be investigated

593 further in the future. This will be done in a forthcoming study by creating mathematical models

594 for the TCS/PR cascade architectures identified in this study and comparing the dynamic 595 behavior of each of the alternatives. 
597

Alm E, Huang K, Arkin A. 2006. The evolution of two-component systems in bacteria reveals different strategies for niche adaptation. PLoS computational biology 2:e143.

Altschul SF, Gish W, Miller W, Myers EW, Lipman DJ. 1990. Basic local alignment search tool. Journal of molecular biology 215:403-10.

Alves R, Savageau MA. 2003. Comparative analysis of prototype two-component systems with either bifunctional or monofunctional sensors: differences in molecular structure and physiological function. Molecular Microbiology 48:25-51.

Appleby JL, Parkinson JS, Bourret RB. 1996. Signal transduction via the multi-step phosphorelay: not necessarily a road less traveled. Cell 86:845-8.

Attwood P V. 2013. Histidine kinases from bacteria to humans. Biochemical Society transactions 41:1023-8.

Bekker M, Teixeira de Mattos MJ, Hellingwerf KJ. 2006. The role of two-component regulation systems in the physiology of the bacterial cell. Science progress 89:213-42.

Blumenthal T, Davis P, Garrido-Lecca A. 2015. Operon and non-operon gene clusters in the C. elegans genome. WormBook: the online review of C. elegans biology:1-20.

Bock A, Gross R. 2002. The unorthodox histidine kinases BvgS and EvgS are responsive to the oxidation status of a quinone electron carrier. European journal of biochemistry / FEBS 269:3479-84.

Bordi C, Théraulaz L, Méjean V, Jourlin-Castelli C. 2003. Anticipating an alkaline stress through the Tor phosphorelay system in Escherichia coli. Molecular microbiology 48:21123.

Buelow DR, Raivio TL. 2010. Three (and more) component regulatory systems - auxiliary regulators of bacterial histidine kinases. Molecular microbiology 75:547-66.

Burbulys D, Trach KA, Hoch JA. 1991. Initiation of sporulation in B. subtilis is controlled by a multicomponent phosphorelay. Cell 64:545-52.

Cağatay T, Turcotte M, Elowitz MB, Garcia-Ojalvo J, Süel GM. 2009. Architecture-dependent noise discriminates functionally analogous differentiation circuits. Cell 139:512-22.

Capra EJ, Laub MT. 2012. Evolution of two-component signal transduction systems. Annual review of microbiology $66: 325-47$. 
626 Casino P, Rubio V, Marina A. 2010. The mechanism of signal transduction by two-component 627 systems. Current opinion in structural biology 20:763-71.

628 Catlett NL, Yoder OC, Turgeon BG. 2003. Whole-genome analysis of two-component signal 629 transduction genes in fungal pathogens. Eukaryotic cell 2:1151-61.

630 Chang C, Kwok SF, Bleecker AB, Meyerowitz EM. 1993. Arabidopsis ethylene-response gene

631

632 ETR1: similarity of product to two-component regulators. Science (New York, N.Y.) 262:539-44.

Clore GM, Venditti V. 2013. Structure, dynamics and biophysics of the cytoplasmic proteinprotein complexes of the bacterial phosphoenolpyruvate: sugar phosphotransferase system. Trends in biochemical sciences 38:515-30.

Cock PJA, Whitworth DE. 2007. Evolution of prokaryotic two-component system signaling pathways: gene fusions and fissions. Molecular biology and evolution 24:2355-7.

Durrens P, Nikolski M, Sherman D. 2008. Fusion and fission of genes define a metric between fungal genomes. PLoS computational biology 4:e1000200.

Eswaramoorthy P, Dinh J, Duan D, Igoshin OA, Fujita M. 2010. Single-cell measurement of the levels and distributions of the phosphorelay components in a population of sporulating Bacillus subtilis cells. Microbiology (Reading, England) 156:2294-304.

Fassler JS, West AH. 2013. Histidine phosphotransfer proteins in fungal two-component signal transduction pathways. Eukaryotic cell 12:1052-60.

Finn RD, Bateman A, Clements J, Coggill P, Eberhardt RY, Eddy SR, Heger A, Hetherington K, Holm L, Mistry J, Sonnhammer ELL, Tate J, Punta M. 2014. Pfam: the protein families database. Nucleic acids research 42:D222-30.

Freeman JA, Bassler BL. 1999. Sequence and function of LuxU: a two-component phosphorelay protein that regulates quorum sensing in Vibrio harveyi. Journal of bacteriology 181:899906.

Galperin MY. 2010. Diversity of structure and function of response regulator output domains.

Galperin MY, Higdon R, Kolker E. 2010. Interplay of heritage and habitat in the distribution of bacterial signal transduction systems. Molecular bioSystems 6:721-8. 
659 Georgellis D, Lynch AS, Lin EC. 1997. In vitro phosphorylation study of the arc two-component 660 signal transduction system of Escherichia coli. Journal of bacteriology 179:5429-35.

661 Gross R, Beier D. 2012. Two-component systems in bacteria. Norfolk UK: Caister Academic 662 Press.

663 Hazelbauer GL, Lai W-C. 2010. Bacterial chemoreceptors: providing enhanced features to two664 component signaling. Current opinion in microbiology 13:124-32.

665

666

667

668

669

670

671

672

673

674

675

676

677

678

679

680

681

682

683

684

685

686

687

688

689
Hoch, J. A., Silhavy TJ. 1995. Two-Component Signal Transduction. Washington, D.C.: American Society for Microbiology.

Huergo LF, Pedrosa FO, Muller-Santos M, Chubatsu LS, Monteiro RA, Merrick M, Souza EM. 2012. PII signal transduction proteins: pivotal players in post-translational control of nitrogenase activity. Microbiology (Reading, England) 158:176-90.

Igoshin OA, Goldbeter A, Kaiser D, Oster G. 2004. A biochemical oscillator explains several aspects of Myxococcus xanthus behavior during development. Proceedings of the National Academy of Sciences of the United States of America 101:15760-5.

Igoshin OA, Alves R, Savageau MA. 2008. Hysteretic and graded responses in bacterial twocomponent signal transduction. Molecular Microbiology 68:1196-1215.

Inouye M, Dutta R. 2003. Histidine kinases in signal transduction. Amsterdam ; Boston: Academic Press.

Jenal U, Galperin MY. 2009. Single domain response regulators: molecular switches with emerging roles in cell organization and dynamics. Current opinion in microbiology 12:15260.

Johnson LS, Eddy SR, Portugaly E. 2010. Hidden Markov model speed heuristic and iterative HMM search procedure. BMC bioinformatics 11:431.

Jones CW, Armitage JP. 2015. Positioning of bacterial chemoreceptors. Trends in microbiology $23: 247-256$.

Jung K, Fried L, Behr S, Heermann R. 2012. Histidine kinases and response regulators in networks. Current opinion in microbiology 15:118-24.

Kim S, Hirakawa H, Muta S, Kuhara S. 2010. Identification and classification of a twocomponent system based on domain structures in bacteria and differences in domain structure between Gram-positive and Gram-negative bacteria. Bioscience, biotechnology, and biochemistry 74:716-20. 
690

691

692

693

694

695

696

697

698

699

700

701

702

703

704

705

706

707

708

709

710

711

712

713

714

715

716

717

718

719

720

721

722

Kobir A, Shi L, Boskovic A, Grangeasse C, Franjevic D, Mijakovic I. 2011. Protein phosphorylation in bacterial signal transduction. Biochimica et biophysica acta 1810:98994.

Krell T, Lacal J, Busch A, Silva-Jiménez H, Guazzaroni M-E, Ramos JL. 2010. Bacterial sensor kinases: diversity in the recognition of environmental signals. Annual review of microbiology 64:539-59.

Kuchina A, Espinar L, Çağatay T, Balbin AO, Zhang F, Alvarado A, Garcia-Ojalvo J, Süel GM. 2011a. Temporal competition between differentiation programs determines cell fate choice. Molecular systems biology 7:557.

Kuchina A, Espinar L, Garcia-Ojalvo J, Süel GM. 2011b. Reversible and noisy progression towards a commitment point enables adaptable and reliable cellular decision-making. PLoS computational biology 7:e1002273.

Laub MT, Goulian M. 2007. Specificity in two-component signal transduction pathways. Annual review of genetics 41:121-45.

Maeda T, Wurgler-Murphy SM, Saito H. 1994. A two-component system that regulates an osmosensing MAP kinase cascade in yeast. Nature 369:242-5.

Majdalani N, Gottesman S. 2005. The Rcs phosphorelay: a complex signal transduction system. Annual review of microbiology 59:379-405.

Mascher T. 2014. Bacterial (intramembrane-sensing) histidine kinases: signal transfer rather than stimulus perception. Trends in microbiology.

Narula J, Devi SN, Fujita M, Igoshin OA. 2012. Ultrasensitivity of the Bacillus subtilis sporulation decision. Proceedings of the National Academy of Sciences of the United States of America 109:E3513-22.

Oka A, Sakai H, Iwakoshi S. 2002. His-Asp phosphorelay signal transduction in higher plants: receptors and response regulators for cytokinin signaling in Arabidopsis thaliana. Genes \& genetic systems 77:383-91.

Ortet P, Whitworth DE, Santaella C, Achouak W, Barakat M. 2015. P2CS: updates of the prokaryotic two-component systems database. Nucleic acids research 43:D536-41.

Ortiz de Orué Lucana D, Groves MR. 2009. The three-component signalling system HbpS-SenSSenR as an example of a redox sensing pathway in bacteria. Amino acids 37:479-86.

Parkinson JS. 1993. Signal transduction schemes of bacteria. Cell 73:857-71.

Perry J, Koteva K, Wright G. 2011. Receptor domains of two-component signal transduction systems. Molecular bioSystems 7:1388-98. 
723

724

725

726

727

728

729

730

731

732

733

734

735

736

737

738

739

740

741

742

743

744

745

746

747

748

749

750

751

752

753

754

Podgornaia AI, Laub MT. 2013. Determinants of specificity in two-component signal transduction. Current Opinion in Microbiology 16:156-162.

Porter SL, Wadhams GH, Armitage JP. 2011. Signal processing in complex chemotaxis pathways. Nature reviews. Microbiology 9:153-65.

Price MN, Arkin AP, Alm EJ. 2006. The life-cycle of operons. PLoS genetics 2:e96.

Ray JCJ, Igoshin OA. 2010. Adaptable functionality of transcriptional feedback in bacterial twocomponent systems. PLoS computational biology 6:e1000676.

Ray JCJ, Igoshin OA. 2012. Interplay of gene expression noise and ultrasensitive dynamics affects bacterial operon organization. PLoS computational biology 8:e1002672.

Ray JCJ, Tabor JJ, Igoshin OA. 2011. Non-transcriptional regulatory processes shape transcriptional network dynamics. Nature reviews. Microbiology 9:817-28.

Sahu SN, Acharya S, Tuminaro H, Patel I, Dudley K, LeClerc JE, Cebula TA, Mukhopadhyay S. 2003. The bacterial adaptive response gene, barA, encodes a novel conserved histidine kinase regulatory switch for adaptation and modulation of metabolism in Escherichia coli. Molecular and cellular biochemistry 253:167-77.

Salvado B, Vilaprinyo E, Karathia H, Sorribas A, Alves R. 2012. Two component systems: physiological effect of a third component. PLoS ONE 7:e31095.

Schaller GE, Shiu S-H, Armitage JP. 2011. Two-component systems and their co-option for eukaryotic signal transduction. Current biology: CB 21:R320-30.

Seshasayee ASN, Luscombe NM. 2011. Comparative genomics suggests differential deployment of linear and branched signaling across bacteria. Molecular bioSystems 7:3042-9.

Sheng X, Huvet M, Pinney JW, Stumpf MPH. 2012. Evolutionary characteristics of bacterial two-component systems. Advances in experimental medicine and biology 751:121-37.

Silva-Jiménez H, Ramos JL, Krell T. 2012. Construction of a prototype two-component system from the phosphorelay system TodS/TodT. Protein engineering, design \& selection : PEDS 25:159-69.

Simon MI, Crane BR, Crane A. 2007. Two-component signaling systems. Amsterdam ; Boston: Elsevier/Academic Press.

Süel GM, Garcia-Ojalvo J, Liberman LM, Elowitz MB. 2006. An excitable gene regulatory circuit induces transient cellular differentiation. Nature 440:545-50.

Swain PS. 2004. Efficient attenuation of stochasticity in gene expression through posttranscriptional control. Journal of molecular biology 344:965-76. 
755

Szurmant H, Hoch JA. 2010. Interaction fidelity in two-component signaling. Current opinion in microbiology 13:190-7.

Szurmant H, White RA, Hoch JA. 2007. Sensor complexes regulating two-component signal transduction. Current opinion in structural biology 17:706-15.

Takeda S, Fujisawa Y, Matsubara M, Aiba H, Mizuno T. 2001. A novel feature of the multistep phosphorelay in Escherichia coli: a revised model of the RcsC --> YojN --> RcsB signalling pathway implicated in capsular synthesis and swarming behaviour. Molecular microbiology 40:440-50.

Thomason P, Kay R. 2000. Eukaryotic signal transduction via histidine-aspartate phosphorelay. Journal of cell science 113 ( Pt 1:3141-50.

Tiwari A, Balázsi G, Gennaro ML, Igoshin OA. 2010. The interplay of multiple feedback loops with post-translational kinetics results in bistability of mycobacterial stress response. Physical biology 7:036005.

Tiwari A, Igoshin OA. 2012. Coupling between feedback loops in autoregulatory networks affects bistability range, open-loop gain and switching times. Physical biology 9:055003.

Uhl MA, Miller JF. 1996. Central role of the BvgS receiver as a phosphorylated intermediate in a complex two-component phosphorelay. The Journal of biological chemistry 271:33176-80.

Ulrich LE, Koonin E V, Zhulin IB. 2005. One-component systems dominate signal transduction in prokaryotes. Trends in microbiology 13:52-6.

Ulrich LE, Zhulin IB. 2010. The MiST2 database: a comprehensive genomics resource on microbial signal transduction. Nucleic acids research 38:D401-7.

Whitworth DE, Cock PJA. 2009. Evolution of prokaryotic two-component systems: insights from comparative genomics. Amino acids 37:459-66.

Williams RHN, Whitworth DE. 2010. The genetic organisation of prokaryotic two-component system signalling pathways. BMC genomics 11:720.

Wolanin PM, Thomason PA, Stock JB. 2002. Histidine protein kinases: key signal transducers outside the animal kingdom. Genome biology 3:REVIEWS3013.

Wolfram S. 1999. The MATHEMATICA ${ }^{\circledR}$ Book, Version 4. Cambridge University Press.

Wuichet K, Cantwell BJ, Zhulin IB. 2010. Evolution and phyletic distribution of two-component signal transduction systems. Current opinion in microbiology 13:219-25. 
786

787

Peer] reviewing PDF | (2015:03:4438:1:1:NEW 30 Jun 2015) 
Figure 1.Two component systems. A- Prototypical two component systems with one phosphotransfer step between HK and RR. B - 3-step phosphorelay, with four protein domains involved in the signal transduction process and 3 phosphotransfer steps.

791

Figure 2.Four different patterns of covalent linkage between the protein domains involved in phosphorelays. A - A four protein phosphorelays. B - A phosphorelay with and hybrid kinase at the beginning of the cascade. $C-A$ two protein phosphorelay where the first two phosphotransfer steps between domains contained in a single protein. D - A one protein phosphorelay, where all phosphotransfer steps take place between domains of a single protein.

Figure 3.Percentage of TCS/PR proteins in the proteome per phylum. The colored box represents the range of percentage values comprised between the $25 \%$ and the $75 \%$ quantiles, and the edges of the vertical bar denote the upper and lower percentage values for each phylum. Phylum abbreviations are given in Table 1. Phyla with only one species surveyed are not represented in the figure. Their percentage of TCS/PR proteins per phylum are: $\mathrm{Ar}(0.93), \mathrm{Cd}(0.89), \mathrm{L}(0.68), \mathrm{Cr}(3.73), \mathrm{El}(0.78), \mathrm{Fb}$ (0.81), Ge (3.15), Z (2.47), Ni (1.97), K (0), N (0), Nh (0), Am (0.17) and M (0.04). We have found only 2 TCS/PR proteins in Av (5 sp): $1 \mathrm{HPt}$ in T. annulata and $1 \mathrm{HK}$ in T. parva.

805

806

Figure 4.Percentage of TCS/PR proteins in the proteome versus total number of proteins in the proteome. The graph on the left depicts the data from prokaryotes, and the graph on the right depicts the data from eukaryotes. $\mathrm{R}^{2}$ is 0.21 for prokaryotes and 0.49 for eukaryotes. This means that proteome size explains $21 \%$ of the variation in the percentage of TCS/PR in prokaryotes and $49 \%$ in

811 eukaryotes.

812

813

814

815

816

817

818

819

820

821

822

823

824 
825 Supplementary Figure 1. Ratio between the observed and the randomly expected frequency 826 of HK genes located next to RR genes in the genome. Phylum abbreviations are explained in Table

827 1. The colored box represents the range of percentage values comprised between the $25 \%$ and the $75 \%$ 828 quantiles, and the edges of the vertical bar denote the upper and lower percentage values for each 829 phylum.

830

831

832

833 
1

Two component systems.

A - Prototypical two component systems with one phosphotransfer step between HK and RR. B - 3-step phosphorelay, with four protein domains involved in the signal transduction process and 3 phosphotransfer steps.

A

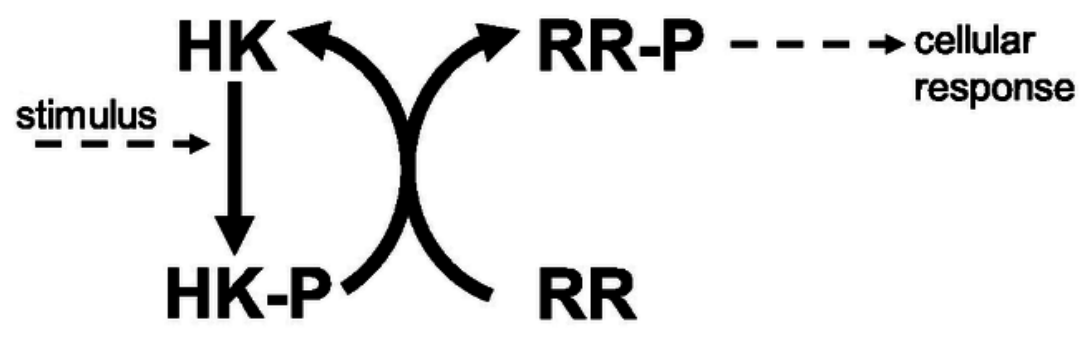

B

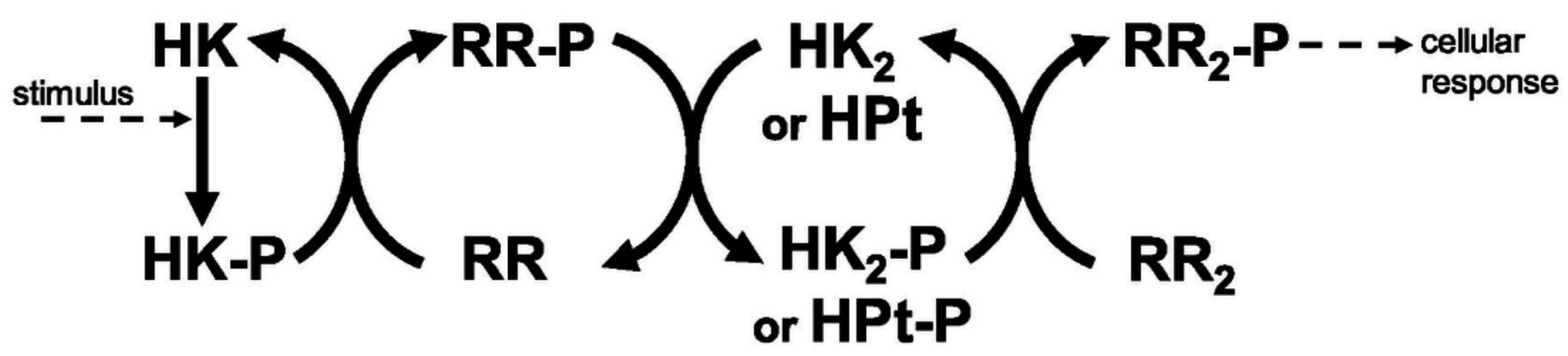


2

Four different patterns of covalent linkage between the protein domains involved in phosphorelays.

A - A four protein phosphorelays. B - A phosphorelay with and hybrid kinase at the beginning of the cascade. C - A two protein phosphorelay where the first two phosphotransfer steps between domains contained in a single protein. D - A one protein phosphorelay, where all phosphotransfer steps take place between domains of a single protein.

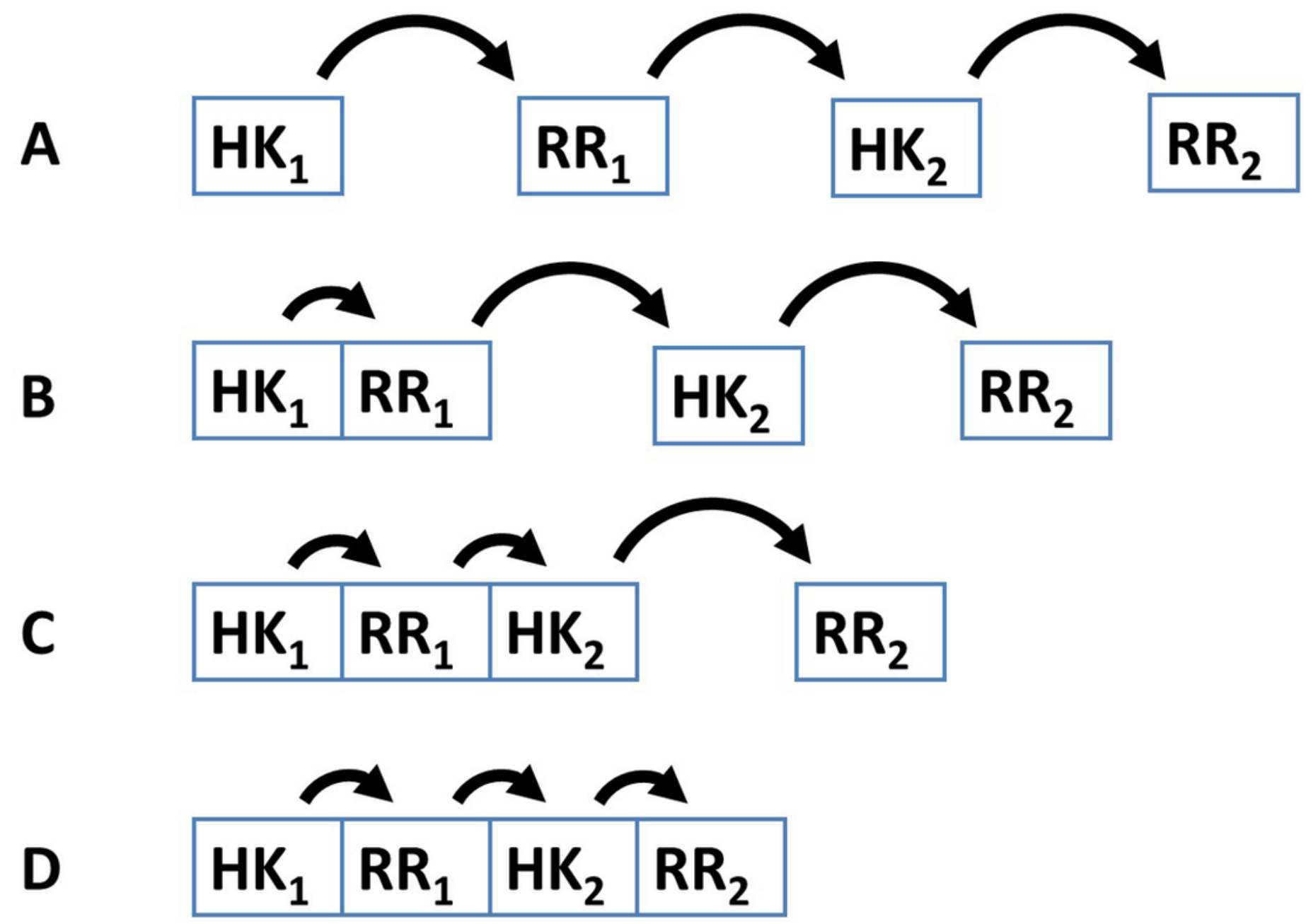




\section{3}

Percentage of TCS/PR proteins in the proteome per phylum.

The colored box represents the range of percentage values comprised between the $25 \%$ and the $75 \%$ quantiles, and the edges of the vertical bar denote the upper and lower percentage values for each phylum. Phylum abbreviations are given in Table 1. Phyla with only one species surveyed are not represented in the figure. Their percentage of TCS/PR proteins per phylum are: Aq (1.30), Ge (3.15), Fb (0.81), L (0.68), Cr (3.73), El (0.78), $\operatorname{Ar}(0.93), \mathrm{Z}(2.47), \mathrm{O}$ (4.95), Ni (1.97), K (0), N (0), Nh (0), Am (0.17) and M (0.04). We have found only 2 TCS/PR proteins in Av (5 sp): $1 \mathrm{HPt}$ in T.annulata and $1 \mathrm{HK}$ in T. parva.

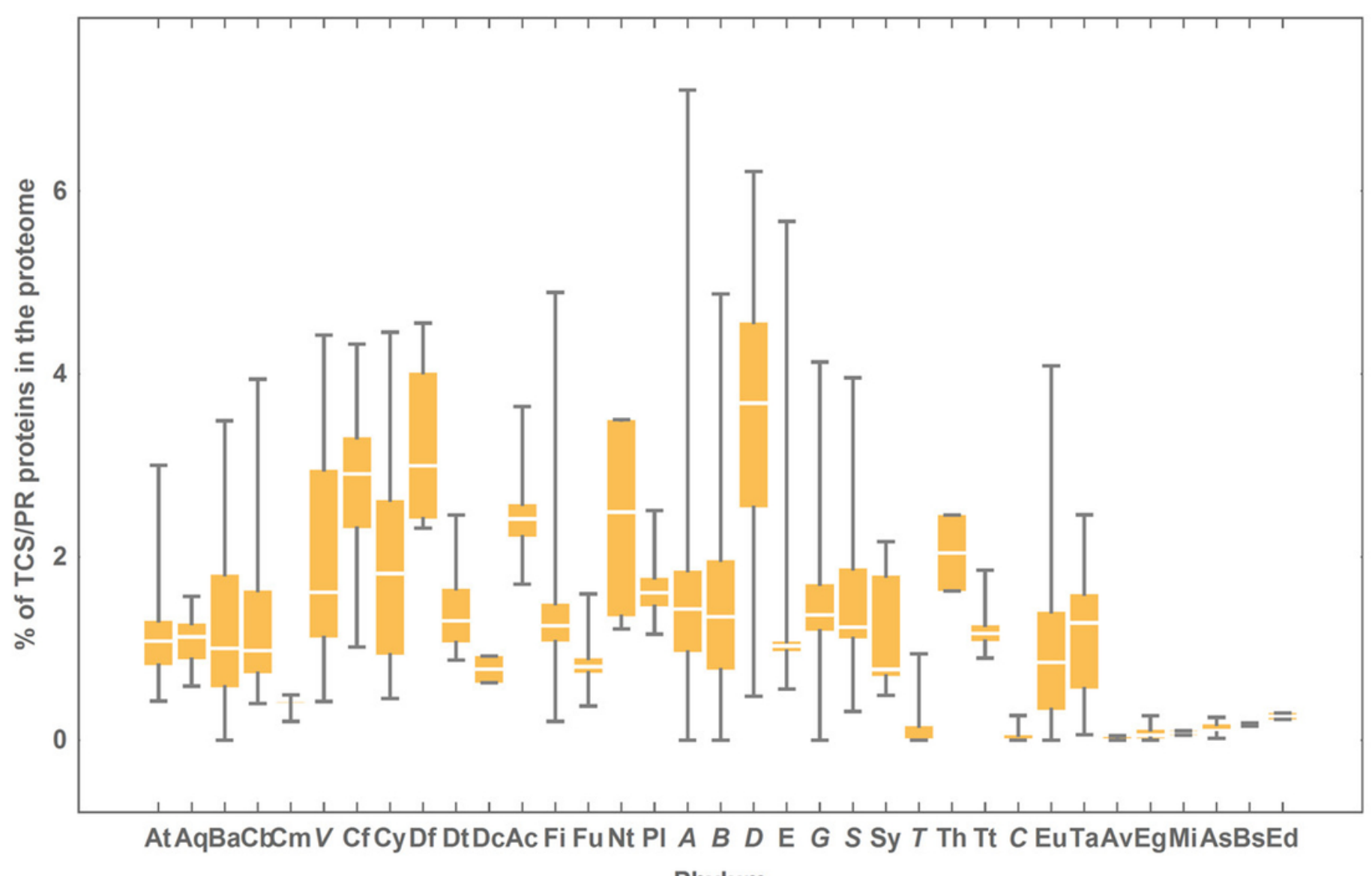

Phylum 
4

Percentage of TCS/PR proteins in the proteome versus total number of proteins in the proteome.

$\mathrm{R}^{2}$ is 0.21 for prokaryotes and 0.49 for eukaryotes. This means that proteome size explains $21 \%$ of the variation in the percentage of TCS/PR in prokaryotes and $49 \%$ in eukaryotes.
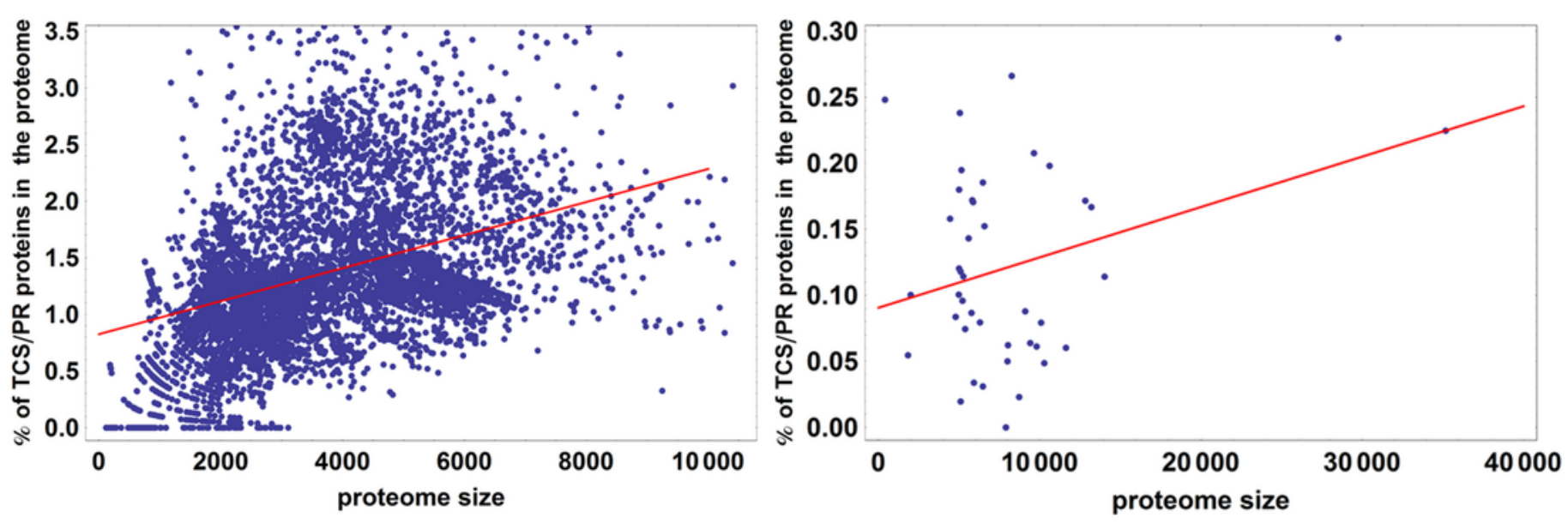


\section{Table 1 (on next page)}

Percentage of species in each phylum with TCS/PR proteins. 
1 Table 1. Percentage of species in each phylum with TCS/PR proteins.

\begin{tabular}{|c|c|c|c|c|c|}
\hline Domain & Phylum & Abbreviaton & $\begin{array}{c}\text { no of } \\
\text { species } \\
\text { surveyed }\end{array}$ & $\begin{array}{c}\text { \% of species } \\
\text { with } \\
\text { HK and RR } \\
\text { domains }\end{array}$ & $\begin{array}{c}\text { \% of species } \\
\text { with HPt } \\
\text { domains }\end{array}$ \\
\hline Bacteria & Actinobacteria & At & 635 & 99.37 & 14.49 \\
\hline Bacteria & Aquificae & $\mathrm{Aq}$ & 13 & 100.00 & 76.92 \\
\hline Bacteria & Armatimonadetes & $\mathrm{Ar}$ & 1 & 100.00 & 100.00 \\
\hline Bacteria & Bacteroidetes & $\mathrm{Ba}$ & 235 & 89.79 & 49.79 \\
\hline Bacteria & Chlorobi & $\mathrm{Cb}$ & 14 & 100.00 & 71.43 \\
\hline Bacteria & Caldiserica & $\mathrm{Cd}$ & 1 & 100.00 & 0.00 \\
\hline Bacteria & Chlamydiae & $\mathrm{Cm}$ & 108 & 98.15 & 1.85 \\
\hline Bacteria & Lentisphaerae & $\mathrm{L}$ & 1 & 100.00 & 100.00 \\
\hline Bacteria & Verrucomicrobia & $\mathrm{V}$ & 10 & 100.00 & 80.00 \\
\hline Bacteria & Chloroflexi & $\mathrm{Cf}$ & 23 & 100.00 & 65.21 \\
\hline Bacteria & Chrysiogenetes & $\mathrm{Cr}$ & 1 & 100.00 & 100.00 \\
\hline Bacteria & Cyanobacteria & $\mathrm{Cy}$ & 118 & 100.00 & 75.42 \\
\hline Bacteria & Deferribacteres & Df & 4 & 100.00 & 100.00 \\
\hline Bacteria & Deinococcus-Thermus & $\mathrm{Dt}$ & 20 & 100.00 & 35.00 \\
\hline Bacteria & Dictyoglomi & Dc & 2 & 100.00 & 0.00 \\
\hline Bacteria & Elusimicrobia & El & 1 & 100.00 & 0.00 \\
\hline Bacteria & Acidobacteria & $A c$ & 9 & 100.00 & 100.00 \\
\hline Bacteria & Fibrobacteres & $\mathrm{Fb}$ & 1 & 100.00 & 100.00 \\
\hline Bacteria & Firmicutes & $\mathrm{Fi}$ & 2066 & 99.42 & 37.80 \\
\hline Bacteria & Fusobacteria & $\mathrm{Fu}$ & 38 & 94.74 & 28.95 \\
\hline Bacteria & Gemmatimonadetes & $\mathrm{Ge}$ & 1 & 100.00 & 100.00 \\
\hline Bacteria & Nitrospinae & $\mathrm{Ni}$ & 1 & 100.00 & 100.00 \\
\hline Bacteria & Nitrospirae & $\mathrm{Nt}$ & 4 & 100.00 & 100.00 \\
\hline Bacteria & Planctomycetes & $\mathrm{PI}$ & 20 & 100.00 & 100.00 \\
\hline Bacteria & Alphaproteobacteria & $A$ & 451 & 96.67 & 58.31 \\
\hline Bacteria & Betaproteobacteria & B & 366 & 98.36 & 59.56 \\
\hline Bacteria & Deltaproteobacteria & $\mathrm{D}$ & 82 & 98.78 & 98.78 \\
\hline Bacteria & Epsilonproteobacteria & $\mathrm{E}$ & 410 & 100.00 & 98.54 \\
\hline Bacteria & Gammaproteobacteria & G & 2246 & 98.31 & 95.46 \\
\hline Bacteria & Zetaproteobacteria & Z & 1 & 100.00 & 100.00 \\
\hline Bacteria & Spirochaetes & $\mathrm{S}$ & 274 & 100.00 & 99.64 \\
\hline Bacteria & Synergistetes & Sy & 11 & 90.91 & 63.64 \\
\hline Bacteria & Tenericutes & $\mathrm{T}$ & 111 & 15.32 & 7.21 \\
\hline Bacteria & Thermodesulfobacteria & Th & 2 & 100.00 & 100.00 \\
\hline Bacteria & Thermotogae & $\mathrm{Tt}$ & 17 & 100.00 & 100.00 \\
\hline Archaea & Crenarchaeota & $\mathrm{C}$ & 51 & 3.92 & 3.92 \\
\hline Archaea & Euryarchaeota & $\mathrm{Eu}$ & 179 & 86.03 & 64.25 \\
\hline Archaea & Korarchaeota & $\mathrm{K}$ & 1 & 0.00 & 0.00 \\
\hline Archaea & Thaumarchaeota & $\mathrm{Ta}$ & 11 & 81.82 & 63.64 \\
\hline Archaea & Nanoarchaeota & $\mathrm{N}$ & 1 & 0.00 & 0.00 \\
\hline Archaea & Nanohaloarchaeota & $\mathrm{Nh}$ & 1 & 0.00 & 0.00 \\
\hline Eukarya & Alveolates & Av & 5 & 0.00 & 20.00 \\
\hline Eukarya & Amoeboflagellates & Am & 1 & 100.00 & 100.00 \\
\hline Eukarya & Euglenozoa & $\mathrm{Eg}$ & 5 & 40.00 & 0.00 \\
\hline Eukarya & Microsporidians & $\mathrm{Mi}$ & 2 & 50.00 & 0.00 \\
\hline Eukarya & Ascomycetes & As & 31 & 54.84 & 96.77 \\
\hline Eukarya & Basidiomycetes & Bs & 2 & 100.00 & 100.00 \\
\hline Eukarya & Eudicots & $\mathrm{Ed}$ & 2 & 100.00 & 100.00 \\
\hline Eukarya & Monocots & $\mathrm{M}$ & 1 & 0.00 & 100.00 \\
\hline Eukarya & Nematodes & - & 1 & 0.00 & 0.00 \\
\hline Eukarya & Arthropods & - & 7 & 0.00 & 0.00 \\
\hline Eukarya & Chordates & - & 10 & 0.00 & 0.00 \\
\hline
\end{tabular}


Table 2 (on next page)

Types of TCS/PR proteins found in the 7609 surveyed species.

The protein identifier describes the type (HK, HPt, or RR) and number of TCS/PR domains fused in each protein. 
1 Table 2. Types of TCS/PR proteins found in the 7609 surveyed species. The protein identifier describes the type 2 (HK, HPt, or RR) and number of TCS/PR domains fused in each protein.

\begin{tabular}{|c|c|c|c|c|}
\hline Protein type & $\begin{array}{l}\text { Total } \\
\text { number of } \\
\text { proteins } \\
\text { found }\end{array}$ & $\begin{array}{l}\text { Percentage of } \\
\text { proteomes with } \\
\text { this type of } \\
\text { protein }\end{array}$ & $\begin{array}{l}\text { Number of } \\
\text { species with } \\
\text { this type of } \\
\text { protein }\end{array}$ & $\begin{array}{l}\text { Average number of } \\
\text { proteins/organism }\end{array}$ \\
\hline $\mathrm{RR}$ & 219436 & 97,07 & 7386 & 29,71 \\
\hline HK & 151849 & 95,98 & 7303 & 20,79 \\
\hline HKRR & 18383 & 48,57 & 3696 & 4,97 \\
\hline HKRRHPt & 9097 & 40,85 & 3108 & 2,93 \\
\hline HKHPt & 5506 & 41,99 & 3195 & 1,72 \\
\hline $\mathrm{HPt}$ & 3534 & 28,05 & 2134 & 1,66 \\
\hline $\mathrm{RR}_{1} \mathrm{RR}_{2}$ & 2034 & 17,60 & 1339 & 1,52 \\
\hline $\mathrm{HKRR}_{1} \mathrm{RR}_{2}$ & 2017 & 13,59 & 1034 & 1,95 \\
\hline $\mathrm{HKRR}_{1} \mathrm{HPtRR}_{2}$ & 982 & 8,12 & 618 & 1,59 \\
\hline $\mathrm{HK}_{1} \mathrm{RR}_{1} \mathrm{RR}_{2} \mathrm{RR}_{3}$ & 580 & 6,58 & 501 & 1,16 \\
\hline $\mathrm{HK}_{1} \mathrm{HK}_{2}$ & 450 & 4,07 & 310 & 1,45 \\
\hline $\mathrm{HK}_{1} \mathrm{RRHK}_{2}$ & 392 & 3,30 & 251 & 1,56 \\
\hline RRHPt & 312 & 3,47 & 264 & 1,18 \\
\hline $\mathrm{HKRRHPt}_{1} \mathrm{HPt}_{2} \mathrm{HPt}_{3}$ & 141 & 1,85 & 141 & 1,00 \\
\hline $\mathrm{RR}_{1} \mathrm{RR}_{2} \mathrm{HPt}$ & 130 & 1,45 & 110 & 1,18 \\
\hline $\mathrm{HKRRHPt}_{1} \mathrm{HPt}_{2} \mathrm{HPt}_{3} \mathrm{HPt}_{4}$ & 108 & 1,42 & 108 & 1,00 \\
\hline $\mathrm{HK}_{1} \mathrm{RR}_{1} \mathrm{HK}_{2} \mathrm{RR}_{2}$ & 90 & 0,79 & 60 & 1,50 \\
\hline $\mathrm{RR}_{1} \mathrm{RR}_{2} \mathrm{RR}_{3} \mathrm{HPt}$ & 72 & 0,51 & 39 & 1,85 \\
\hline $\mathrm{HKRRHPt}_{1} \mathrm{HPt}_{2} \mathrm{HPt}_{3} \mathrm{HPt}_{4} \mathrm{HPt}_{5}$ & 61 & 0,80 & 61 & 1,00 \\
\hline $\mathrm{HKRRHPt}_{1} \mathrm{HPt}_{2}$ & 58 & 0,72 & 55 & 1,05 \\
\hline $\mathrm{HK}_{1} \mathrm{HK}_{2} \mathrm{RRHPt}$ & 39 & 0,50 & 38 & 1,03 \\
\hline $\mathrm{HK}_{1} \mathrm{HK}_{2} \mathrm{HPt}$ & 39 & 0,50 & 38 & 1,03 \\
\hline $\mathrm{HKHPt}_{1} \mathrm{HPt}_{2}$ & 36 & 0,46 & 35 & 1,03 \\
\hline $\mathrm{RR}_{1} \mathrm{RR}_{2} \mathrm{RR}_{3}$ & 34 & 0,32 & 24 & 1,42 \\
\hline $\mathrm{HKRR}_{1} \mathrm{RR}_{2} \mathrm{RR}_{3} \mathrm{HPt}$ & 33 & 0,37 & 28 & 1,18 \\
\hline $\mathrm{HPt}_{1} \mathrm{HPt}_{2}$ & 21 & 0,20 & 15 & 1,40 \\
\hline $\mathrm{HKHPt}_{1} \mathrm{HPt}_{2} \mathrm{HPt}_{3}$ & 16 & 0,20 & 15 & 1,07 \\
\hline $\mathrm{HK}_{1} \mathrm{HK}_{2} \mathrm{RR}_{1} \mathrm{RR}_{2} \mathrm{RR}_{3}$ & 9 & 0,12 & 9 & 1,00 \\
\hline $\mathrm{HK}_{1} \mathrm{HK}_{2} \mathrm{HK}_{3}$ & 9 & 0,04 & 3 & 3,00 \\
\hline $\mathrm{HKRR}_{1} \mathrm{RR}_{2} \mathrm{RR}_{3} \mathrm{RR}_{4} \mathrm{RR}_{5} \mathrm{HPt}$ & 7 & 0,09 & 7 & 1,00 \\
\hline $\mathrm{HKRRHPt}_{1} \mathrm{HPt}_{2} \mathrm{HPt}_{3} \mathrm{HPt}_{4} \mathrm{HPt}_{5} \mathrm{HPt}_{6} \mathrm{HPt}_{7}$ & 7 & 0,09 & 7 & 1,00 \\
\hline $\mathrm{HKRR}_{1} \mathrm{RR}_{2} \mathrm{RR}_{3} \mathrm{RR}_{4}$ & 6 & 0,08 & 6 & 1,00 \\
\hline $\mathrm{HK}_{1} \mathrm{HK}_{2} \mathrm{HK}_{3} \mathrm{HK}_{4} \mathrm{RR}_{1} \mathrm{RR}_{2}$ & 6 & 0,08 & 6 & 1,00 \\
\hline $\mathrm{HK}_{1} \mathrm{HK}_{2} \mathrm{RRHPt}_{1} \mathrm{HPt}_{2}$ & 5 & 0,07 & 5 & 1,00 \\
\hline $\mathrm{HKRR}_{1} \mathrm{RR}_{2} \mathrm{RR}_{3} \mathrm{RR}_{4} \mathrm{HPt}$ & 5 & 0,07 & 5 & 1,00 \\
\hline $\mathrm{RR}_{1} \mathrm{RR}_{2} \mathrm{RR}_{3} \mathrm{RR}_{4}$ & 2 & 0,03 & 2 & 1,00 \\
\hline $\mathrm{HK}_{1} \mathrm{HK}_{2} \mathrm{RR}_{1} \mathrm{RR}_{2} \mathrm{HPt}_{1} \mathrm{HPt}_{2}$ & 2 & 0,03 & 2 & 1,00 \\
\hline $\mathrm{HK}_{1} \mathrm{HK}_{2} \mathrm{RR}_{1} \mathrm{RR}_{2} \mathrm{RR}_{3} \mathrm{RR}_{4}$ & 2 & 0,03 & 2 & 1,00 \\
\hline $\mathrm{HK}_{1} \mathrm{HK}_{2} \mathrm{HK}_{3} \mathrm{HK}_{4}$ & 2 & 0,03 & 2 & 1,00 \\
\hline $\mathrm{HK}_{1} \mathrm{HK}_{2} \mathrm{HPt}_{1} \mathrm{HPt}_{2}$ & 2 & 0,03 & 2 & 1,00 \\
\hline $\mathrm{HKRR}_{1} \mathrm{RR}_{2} \mathrm{HPt}_{1} \mathrm{HPt}_{2}$ & 2 & 0,03 & 2 & 1,00 \\
\hline $\mathrm{HK}_{1} \mathrm{HK}_{2} \mathrm{HK}_{3} \mathrm{RR}$ & 1 & 0,01 & 1 & 1,00 \\
\hline $\mathrm{HPt}_{1} \mathrm{HPt}_{2} \mathrm{HPt}_{3}$ & 1 & 0,01 & 1 & 1,00 \\
\hline $\mathrm{HK}_{1} \mathrm{HK}_{2} \mathrm{RRHPt}_{1} \mathrm{HPt}_{2} \mathrm{HPt}_{3}$ & 1 & 0,01 & 1 & 1,00 \\
\hline $\mathrm{HKRR}_{1} \mathrm{RR}_{2} \mathrm{RR}_{3} \mathrm{HPt}_{1} \mathrm{HPt}_{2} \mathrm{HPt}_{3}$ & 1 & 0,01 & 1 & 1,00 \\
\hline $\mathrm{HPt}_{1} \mathrm{HPt}_{2} \mathrm{HPt}_{3} \mathrm{HPt}_{4}$ & 1 & 0,01 & 1 & 1,00 \\
\hline $\mathrm{HKRR}_{1} \mathrm{RR}_{2} \mathrm{HPt}_{1} \mathrm{HPt}_{2} \mathrm{HPt}_{3}$ & 1 & 0,01 & 1 & 1,00 \\
\hline $\mathrm{HK}_{1} \mathrm{HK}_{2} \mathrm{RR}_{1} \mathrm{RR}_{2} \mathrm{HPt}$ & 1 & 0,01 & 1 & 1,00 \\
\hline $\mathrm{HK}_{1} \mathrm{HK}_{2} \mathrm{RR}_{1} \mathrm{RR}_{2} \mathrm{RR}_{3} \mathrm{RR}_{4} \mathrm{RR}_{5} \mathrm{RR}_{6} \mathrm{HPt}$ & 1 & 0,01 & 1 & 1,00 \\
\hline $\mathrm{HKRRHPt}_{1} \mathrm{HPt}_{2} \mathrm{HPt}_{3} \mathrm{HPt}_{4} \mathrm{HPt}_{5} \mathrm{HPt}_{6}$ & 1 & 0,01 & 1 & 1,00 \\
\hline
\end{tabular}




\section{Table 3(on next page)}

Total number of HKRRHPt and HKRRHK proteins found in prokaryotic phyla.

Phyla in bold are from the bacterial domain. Italicized phyla are from the archaeal domain. 
1 Table 3. Total number of HKRRHPt and HKRRHK proteins found in prokaryotic phyla. Phyla in bold are from the bacterial domain. Italicized phyla are from the 2 archaeal domain.

\begin{tabular}{|c|c|c|c|}
\hline Phylum & $\begin{array}{l}\text { Number of HKRRHPt/HK }{ }_{1} \mathrm{RRHK}_{2} \text { proteins } \\
\text { found }\end{array}$ & $\begin{array}{c}\text { Number of HKRRHPt/HK }{ }_{1} \text { RRHK }_{2} \text { genes } \\
\text { with a neighboring RR gene }\end{array}$ & $\begin{array}{c}\text { \% of HKRRHPt / } \mathrm{HK}_{1} \mathrm{RRHK}_{2} \text { genes with a } \\
\text { neighboring RR gene }\end{array}$ \\
\hline Actinobacteria & $12 / 4$ & $9 / 1$ & $75.00 / 25.00$ \\
\hline Aquificae & $0 / 0$ & $0 / 0$ & $-/-$ \\
\hline Armatimonadetes & $0 / 0$ & $0 / 0$ & $-/-$ \\
\hline Bacteroidetes & $107 / 9$ & $62 / 4$ & $57.94 / 44.44$ \\
\hline Chlorobi & $4 / 0$ & $0 / 0$ & $0.00 /-$ \\
\hline Caldiserica & $0 / 0$ & $0 / 0$ & $-/-$ \\
\hline Chlamydiae & $2 / 0$ & $1 / 0$ & $50.00 /-$ \\
\hline Lentisphaerae & $1 / 0$ & $0 / 0$ & $0.00 /-$ \\
\hline Verrucomicrobia & $12 / 2$ & $9 / 1$ & $75.00 / 50.00$ \\
\hline Chloroflexi & $16 / 0$ & $8 / 0$ & $50.00 /-$ \\
\hline Chrysiogenetes & $1 / 0$ & $0 / 0$ & $0.00 /-$ \\
\hline Cyanobacteria & $193 / 28$ & $41 / 9$ & $21.24 / 32.14$ \\
\hline Deferribacteres & $9 / 0$ & $7 / 0$ & 77.78/- \\
\hline Deinococcus-Thermus & $0 / 4$ & $0 / 1$ & $-/ 25.00$ \\
\hline Dictyoglomi & $0 / 0$ & $0 / 0$ & $-/-$ \\
\hline Elusimicrobia & $0 / 0$ & $0 / 0$ & $-/-$ \\
\hline Acidobacteria & $1 / 5$ & $1 / 2$ & $100.00 / 40.00$ \\
\hline Fibrobacteres & $0 / 0$ & $0 / 0$ & $-/-$ \\
\hline Firmicutes & $65 / 97$ & $44 / 69$ & $67.69 / 71.13$ \\
\hline Fusobacteria & $2 / 0$ & $2 / 0$ & 100.00/- \\
\hline Gemmatimonadetes & $3 / 0$ & $3 / 0$ & 100.00/- \\
\hline Nitrospinae & $0 / 0$ & $0 / 0$ & $-/-$ \\
\hline Nitrospirae & $4 / 0$ & $3 / 0$ & $75.00 /-$ \\
\hline Planctomycetes & $40 / 0$ & $18 / 0$ & $45.00 /-$ \\
\hline Alphaproteobacteria & $337 / 10$ & $233 / 5$ & $69.14 / 50.00$ \\
\hline Betaproteobacteria & $364 / 9$ & $274 / 4$ & $75.27 / 44.44$ \\
\hline Deltaproteobacteria & $208 / 29$ & $131 / 1$ & $62.98 / 3.45$ \\
\hline Epsilonproteobacteria & $399 / 0$ & $389 / 0$ & 97.49/- \\
\hline Gammaproteobacteria & $7239 / 28$ & $3336 / 15$ & $46.08 / 53.57$ \\
\hline Zetaproteobacteria & $2 / 0$ & $1 / 0$ & $50.00 /-$ \\
\hline Spirochaetes & $53 / 147$ & $16 / 3$ & $30.19 / 2.04$ \\
\hline Synergistetes & $6 / 0$ & $6 / 0$ & $100.00 /-$ \\
\hline Tenericutes & $0 / 0$ & $0 / 0$ & $-/-$ \\
\hline Thermodesulfobacteria & $2 / 0$ & $1 / 0$ & $50.00 /-$ \\
\hline Thermotogae & $6 / 0$ & $5 / 0$ & 83.33/- \\
\hline Crenarchaeota & $0 / 0$ & $0 / 0$ & $-/-$ \\
\hline Euryarchaeota & $9 / 1$ & $3 / 0$ & $33.33 / 0.00$ \\
\hline Thaumarchaeota & $0 / 0$ & $0 / 0$ & $-/-$ \\
\hline
\end{tabular}


PeerJ Reviewing Manuscript 


\section{Table 4 (on next page)}

Percentage of RR and HK domains in hybrid proteins as a function of the total number of $H K$ and RR proteins in the genome.

Phyla in bold are from the bacterial domain. Italicized phyla are from the archaeal domain. Other phyla are from the eukaryotic domain. 
1 Table 4. Percentage of RR and HK domains in hybrid proteins as a function of the total number of HK and RR

2 proteins in the genome. Phyla in bold are from the bacterial domain. Italicized phyla are from the archaeal

3 domain. Other phyla are from the eukaryotic domain.

\begin{tabular}{|c|c|c|}
\hline Phylum & RR & SK \\
\hline Gammaproteobacteria & $6.97+0.2 x^{* *}$ & $14+0.31 x^{* *}$ \\
\hline Betaproteobacteria & $1.90+0.22 x^{* *}$ & $4.6+0.37 x^{* *}$ \\
\hline Epsilonproteobacteria & $15.3-0.06 x^{+}$ & $0.17+0.36 x^{*}$ \\
\hline Deltaproteobacteria & $17.3+0.1 x^{*}$ & $31.9+0.06 x^{+}$ \\
\hline Alphaproteobacteria & $4.1+0.29 x^{* *}$ & $3.8+0.4 x^{* *}$ \\
\hline Firmicutes & $-0.6+0.1 x^{* *}$ & $1.7+0.09 x^{*}$ \\
\hline Tenericutes & -- & --- \\
\hline Actinobacteria & $-4.5+0.32 x^{* *}$ & $-0.38+0.2 x^{*}$ \\
\hline Chlamydiae & --- & --- \\
\hline Spirochaetes & $5+0.47 x^{*}$ & $27.2+0.07 x^{+}$ \\
\hline Acidobacteria & $-5.7+0.26 x$ & $-16+0.53 x^{*}$ \\
\hline Bacteroidetes & $30.7+0.05 x^{+}$ & $32+0.09 x^{+}$ \\
\hline Fusobacteria & $-5.8+x$ & $-7.1+1.5 x$ \\
\hline Verrumicrobia & $6.7+0.3 x^{+}$ & $6.6+0.4 x^{+}$ \\
\hline Planctomycetes & $32.8-0.1 x^{+}$ & $49.8-0.21 x^{+}$ \\
\hline Synergistetes & --- & --- \\
\hline Cyanobacteria & $2.2+0.3 x^{* *}$ & $5.8+0.4 x^{* *}$ \\
\hline Green sulfur bacteria & $31.6+0.7 x^{+}$ & $33.5+0.5 x^{+}$ \\
\hline Green non-sulfur bacteria & $5.2+0.2 x$ & $8.9+0.2 x$ \\
\hline Deinococcus-Thermus & $-1.2+0.2 x$ & $-1.6+0.2 x$ \\
\hline Euryarchaeota & $6.9+0.6 x^{*}$ & $15.4+0.1 x^{+}$ \\
\hline Crenarchaeota & --- & --- \\
\hline Nanoarchaeota & --- & --- \\
\hline Korarchaeota & --- & --- \\
\hline Oomycetes & --- & --- \\
\hline Diatoms & --- & --- \\
\hline Parabasilids & --- & --- \\
\hline Diplomonads & --- & --- \\
\hline Euglenozoa & --- & --- \\
\hline Alveolates & --- & $9.5+0.4 x^{+}$ \\
\hline Amoeboflagellates & --- & --- \\
\hline Choanoflagellates & --- & --- \\
\hline Microsporideans & --- & --- \\
\hline Basidiomycetes & $25.7+3.7 x$ & $88+1.1 x^{+}$ \\
\hline Ascomycetes & $37.4+2.5 x^{* *}$ & $92.4-0.07 x^{+}$ \\
\hline Red algae & --- & --- \\
\hline Green algae & $29.2+x^{+}$ & $114.7-9 x^{+}$ \\
\hline Mosses & -- & --- \\
\hline Monocots & $12.3+0.2 x^{+}$ & $32.9+1.9 x^{+}$ \\
\hline Eudicots & $17.4+0.1 x^{+}$ & $71-0.5 x^{+}$ \\
\hline
\end{tabular}

